BULLETIN OF THE

AMERICAN MATHEMATICAL SOCIETY

Volume 79, Number 1, January 1973

\title{
FUNCTIONS OF SEVERAL NONCOMMUTING VARIABLES ${ }^{1}$
}

\section{BY JOSEPH L. TAYLOR ${ }^{2}$}

The notions of nonsingularity, resolvent set, and spectrum, and the corresponding analytic functional calculus for $n$-tuples of elements of a commutative Banach algebra provide some of the deepest and most potent tools of modern analysis. A point of view one can adopt regarding this theory is as follows: The class of algebras $\mathcal{O}(U)$, for $U$ a domain in $\boldsymbol{C}^{n}$, provides a relatively small, well-understood, and nicely behaved class of topological algebras with distinguished tuples $\left(z_{1}, \ldots, z_{n}\right)$ of elements; furthermore, spectral theory and the Shilov-Arens-Calderón Theorem (cf. [17]) give precise information regarding which algebras $\mathcal{O}(U)$ can be mapped into a given commutative Banach algebra (or $F$-algebra) by a continuous homomorphism carrying $\left(z_{1}, \ldots, z_{n}\right)$ onto a specified tuple of elements. Thus, the algebras $\mathcal{O}(U)$ and tuples $\left(z_{1}, \ldots, z_{n}\right)$ provide a tractable class of models for the behavior of $n$-tuples of elements of a commutative topological algebra.

In [7] and [8] we showed how to extend spectral theory and the analytic functional calculus in a well-defined manner to the study of commuting $n$-tuples of operators on a Banach space. From this point of view, the pairs $\left(\mathcal{O}(U),\left(z_{1}, \ldots, z_{n}\right)\right)$ provide models for the behavior of $n$-tuples of operators.

It must occur to nearly every analyst who encounters joint spectral theory to wonder whether or not there are useful notions of nonsingularity and spectrum for tuples in a noncommutative algebra or for noncommuting tuples of operators. From the point of view we have adopted regarding spectral theory, a more meaningful question is the following: Is there a reasonably small, well behaved class of pairs $\left(A,\left(z_{1}, \ldots, z_{n}\right)\right)$, consisting of an algebra $A$ and an $n$-tuple $\left(z_{1}, \ldots, z_{n}\right)$ of elements of $A$, that will serve as models for the behavior of fairly general (noncommutative) $n$-tuples of algebra elements or $n$-tuples of operators? Given such a class, the analogue of spectral theory and the functional calculus would consist of techniques for deciding which models $\left(A,\left(z_{1}, \ldots, z_{n}\right)\right)$ can be mapped into a given algebra $n$-tuple pair $\left(B,\left(a_{1}, \ldots, a_{n}\right)\right)$, or equivalently, which models

An invited address delivered to the 694th Meeting of the Society in Berkeley, on April 22,1972 ; received by the editors May 5, 1972.

AMS (MOS) subject classifications (1969). Primary 4650; Secondary 4730.

Key words and phrases. Topological algebras, free algebras, localizations, operators, analytic functions.

${ }^{1}$ Research partially supported by the United States Air Force, Air Force Office of Scientific Research, under AFOSR grant 1313-67.

${ }^{2}$ The author is a fellow of the Alfred P. Sloan Foundation. 
$\left(A,\left(z_{1}, \ldots, z_{n}\right)\right)$ have representations on a given space $X$ for which the elements $z_{1}, \ldots, z_{n}$ act as given operators $a_{1}, \ldots, a_{n} \in L(X)$.

The above question has intrigued us for some time. Our definition in [7] of joint spectrum for commuting $n$-tuples of operators can be phrased in terms of concepts from homological algebra which make perfectly good sense in noncommutative situations (cf. [9]); that is, our version of commutative joint spectral theory can be viewed as the study of certain homological relations between modules over the $n$-variable polynomial algebra $P_{n}$; and these are relations which also make sense for modules over more general (even noncommutative) algebras. This suggested that homological algebra might provide the key to defining and studying a class of models for the noncommutative situation. The pursuit of this idea led to a paper [10] on homology and cohomology for topological algebras, and another [11] applying this work to obtain a version of spectral theory which makes sense in a wide variety of situations (both commutative and noncommutative). Here we shall review those portions of this latter paper (with the homological algebra suppressed) that relate directly to the study of noncommutative $n$-tuples of operators, and also present some more recent results on the subject.

In order to develop a class of models $\left(A,\left(z_{1}, \ldots, z_{n}\right)\right)$ for the behavior of noncommutative $n$-tuples, we begin with the free algebra $F_{n}$ on $n$ generators $z_{1}, \ldots, z_{n}$ and identify certain embeddings $F_{n} \rightarrow A$ of $F_{n}$ in "satellite" algebras $A$. These algebras are to be in some sense algebras of functions of the free variables $z_{1}, \ldots, z_{n}$-just as the algebras $\mathcal{O}(U)$ are algebras of functions of the generators of the polynomial algebra $P_{n}$.

A brief discussion of the single variable functional calculus may be helpful in understanding what we shall be doing. Thus, let $a \in L(X)$ be a bounded linear operator on the Banach space $X$. Then $a$ determines an algebra homomorphism $p \rightarrow p(a)$ from the polynomial algebra $P$ into the algebra $L(X)$, that is, a representation of $P$ on $X$ (or a $P$-module structure on $X$ ). Now the algebra $P$ is canonically embedded in each of the algebras $\mathcal{O}(U)$ (the algebra of all holomorphic functions on $U$ ) for $U$ a domain in $C$. Hence, we can ask the question: For which algebras $\mathcal{O}(U)$ does the representation of $P$ on $X$ determined by $a$ extend to a (continuous) representation of $\mathcal{O}(U)$ on $X$ ? Of course, the answer is supplied by the analytic functional calculus: The representation extends to $\mathcal{O}(U)$ if and only if $U$ contains the spectrum of the operator $a$; furthermore, the extension is unique if it exists.

The fact that the above question has such a nice answer is no accident. It is due to the fact that there is a very special relationship between the algebra $P$ and the algebras $\mathcal{O}(U)$. If $A$ is any topological algebra and $\varphi$ a representation of $A$ on an 1.c.s. $X$, one could ask the question: For which 
embeddings, $A \rightarrow B$, of $A$ in other topological algebras, $B$, is it true that the representation extends uniquely to a representation of $B$ ? One would not, however, expect such a general question to have a reasonable answer. For a given $A$, however, it might be possible to identify a class of "satellite" algebras $B$ containing $A$ for which the question is tractable for a sufficiently restricted class of representations $\varphi$. This is certainly the case for $P$; the class of "satellite" algebras is the class of algebras $\mathcal{O}(U)$; for these, and the representations of $P$ on Banach spaces, the functional calculus problem has a nice answer.

What is special about the relationship between $P$ and the algebras $\mathcal{O}(U)$ ? In some sense the elements of $\mathcal{O}(U)$ are functions of the generator of $P$. However, there is a more precise statement of this relationship, in homological terms, which can be generalized to other situations. Roughly speaking, an algebra embedding $A \rightarrow B$ is a localization if representations of $A$ which extend to representations of $B$ do so uniquely, and if the homological relations between representations of $B$ ( $B$-modules) are independent of whether the modules are considered $A$-modules or $B$-modules (cf. [11]). Here we are referring to a version of homological algebra developed for topological algebras (cf. [10]).

The idea, then, is to begin with a given "base" algebra $A$ and study its family of "satellite" algebras $B$, i.e., those algebras which arise from localizations $A \rightarrow B$ of $A$. Representations of $A$ are then classified according to which localizations $A \rightarrow B$ have the property that the representation extends to $B$.

For the base algebra $P_{n}$ (the $n$-variable polynomial algebra), each of the natural embeddings $P_{n} \rightarrow \mathcal{O}(U)$, for $U$ a domain of holomorphy in $C^{n}$, is a localization (cf. $[\mathbf{1 1}, \S 6])$. However, these are not the only localizations of $P_{n}$; others include the natural map $P_{n} \rightarrow C^{\infty}(V)$, for $V$ a domain in $R^{n}$, the map $P_{n} \rightarrow \mathscr{E}^{\prime}\left(R^{n}\right)$ of $P_{n}$ into the algebra of compactly supported distributions determined by sending the generators of $P_{n}$ to the partial derivatives of the Dirac $\delta$, and embeddings of $P_{n}$ in algebras of rational functions.

Other base algebras whose localizations are discussed in [11] are the free algebra $F_{n}$ on $n$-generators, the universal algebra of a Lie algebra, and the algebra of compactly supported distributions on a Lie group.

Every $n$-tuple of operators determines a representation of the free algebra $F_{n}$ on $n$-generators. Thus, it is the natural base algebra to use in the study of noncommuting $n$-tuples. The condition that an algebra homomorphism $F_{n} \rightarrow A$ be a localization is equivalent to the existence of certain operators $\Delta_{1}, \ldots, \Delta_{n}$ from $A$ to $A \hat{\otimes} A$ that have properties reminiscent of partial derivatives (cf. $[\mathbf{1 1}, \S 6])$. In this paper we shall restrict our attention to localizations of $F_{n}$. We shall use the existence of 
the operators $\Delta_{1}, \ldots, \Delta_{n}$ to define the concept (rather than using the homological definition of [11]), and to develop several of its implications. There are remarkable similarities between localizations of $F_{n}$ and ordinary algebras of functions of one or more variables; we shall stress these similarities throughout the paper.

In $\S 1$ we introduce localizations of $F_{n}$ and discuss their elementary properties. In $\$ 2$ we begin our discussion of the analytic functional calculus problem, i.e., the problem of deciding for which localizations $F_{n} \rightarrow A$ a given representation of $F_{n}$ extends to a representation of $A$. This discussion is continued later, in $\S 7$, where we introduce a concept related to the resolvent set of ordinary spectral theory and outline a method of using this concept to attack the functional calculus problem; this method actually supplies a new proof of the ordinary single variable analytic functional calculus theorem.

The class of all localizations of $F_{n}$ will prove too large for our purposes. In fact, the localizations of $F_{1}=P$ include many algebras other than the algebras $\mathcal{O}(U)$. Thus, in $\S 3$ we introduce a class of localizations of $F_{n}$ which we call free analytic algebras. These are localizations which are $F$-algebras such that the operators $\Delta_{1}, \ldots, \Delta_{n}$ satisfy certain regularity properties relative to a sequence of seminorms defining the topology of the algebra. In one variable, the free analytic algebras are just the algebras $\mathcal{O}(U)$ for $U$ a domain in $C$.

An algebra homomorphism $F_{n} \rightarrow A$ is determined by specifying the images $z_{1}, \ldots, z_{n}$ of the generators of $F_{n}$. If $F_{n} \rightarrow A$ is a localization (or free analytic algebra) we call $z_{1}, \ldots, z_{n}$ its generators. For a localization $F_{n} \rightarrow A$ with generators $z_{1}, \ldots, z_{n}$, it turns out that each representation of $A$ is determined by its values on $z_{1}, \ldots, z_{n}$. Thus, we will commonly denote a representation of $A$ on $X$ by $u \rightarrow u(\alpha): A \rightarrow L(X)$, where $\alpha=$ $\left(a_{1}, \ldots, a_{n}\right)$ is the image of the tuple $\left(z_{1}, \ldots, z_{n}\right)$; the $a_{i}$ 's will be called the generators of the representation. Free analytic algebras $A$ have a fairly surprising property: If $u \rightarrow u(\alpha): A \rightarrow L(X)$ is a representation of $A$ on a Banach space $X$, then for all $\beta=\left(b_{1}, \ldots, b_{n}\right)$ in a neighborhood of $\alpha=\left(a_{1}, \ldots, a_{n}\right) \in \oplus^{n} L(X)$, there is a representation $u \rightarrow u(\beta)$ of $A$ on $X$ with generators $b_{1}, \ldots, b_{n}$; furthermore, $u \rightarrow u(\beta)$ varies analytically with $\beta$ in this neighborhood (cf. §3). There are even analogues of Taylor's formula and power series expansions for free analytic algebras; these are developed in $\$ 4$.

Examples of free analytic algebras can be obtained by completing $F_{n}$ in the topology generated by a sequence of power series type norms. The resulting algebras are the usual free analogues of power series algebras (cf. §3). However, these are not the only free analytic algebras. In $\S 4$ we describe an analogue of the implicit function theorem which specifies that 
certain sets of relations in a free analytic algebra $A$ generate ideals $I$ such that $A / I$ is a free analytic algebra on fewer generators. This allows one to construct free analytic algebras in which certain elements have inverses or certain equations have solutions.

We conjecture that free analytic algebras must be Montel spaces (bounded sets are precompact). This is certainly true in one variable. In $\$ 5 \mathrm{we}$ present a discussion and partial result on the general problem.

We end this introduction with an apology: Although we feel that the ideas presented here are promising, it is too early to predict whether or not a significant theory will result from further development. We know very little about the class of all free analytic algebras. As yet, we have no significant applications of what we do know. The techniques of $\$ 6$ for attacking the functional calculus problem might prove to be practical, for $n>1$, only in cases where the ensuing results were trivial anyway. Still, we feel quite strongly that free analytic algebras are the "correct" free analogues of algebras of analytic functions, and that this justifies pursuing the matter for awhile.

1. Localizations of $F_{n}$. Throughout our discussions we shall be dealing with complete, Hausdorff, locally convex algebras over $\boldsymbol{C}$, with identity, in which the multiplication is at least separately continuous. Rather than repeating these conditions ad infinitum, we shall refer to such an object simply as an algebra. A map $A \rightarrow B$ between two algebras will be called a homomorphism if it is an algebraic homomorphism and is continuous; if it maps the identity to the identity, it will be called unital.

The theory of topological tensor products is a central tool for our work (cf. [2], [5], [6], [12]). Given two locally convex topological vector spaces (1.c.s.'s) $X$ and $Y$, we denote the completed inductive tensor product of $X$ and $Y$ by $X \hat{\otimes} Y$. This is a complete 1.c.s. with a separately continuous bilinear map $(x, y) \rightarrow x \otimes y: X \times Y \rightarrow X \hat{\otimes} Y$ such that any separately continuous bilinear map $\varphi: X \times Y \rightarrow Z$, into a complete l.c.s. $Z$, determines a unique continuous linear map $\tilde{\varphi}: X \hat{\otimes} Y \rightarrow Z$ with $\tilde{\varphi}(x \otimes y)$ $=\varphi(x, y)$. Thus, the conditions we have imposed on an algebra $A$ ensure that the multiplication map $A \times A \rightarrow A$ determines a continuous linear $\operatorname{map} \pi: A \hat{\otimes} A \rightarrow A$ with $\pi(u \otimes v)=u v$.

Fur $F$-spaces $X$ and $Y$, separately continuous linear maps $X \times Y \rightarrow Z$ are jointly continuous and the inductive tensor product $X \hat{\otimes} Y$ agrees with the projective tensor product (characterized as above, but with jointly continuous bilinear maps) (cf. [5, III, §5]). Most of the algebras and modules we are interested in are $F$-spaces.

For an algebra $A$, a left $A$-module will be a complete 1.c.s. $X$ together with an associative, separately continuous bilinear map $(u, x) \rightarrow u x$ : $A \times X \rightarrow X$ such that $1 x=x$. This map induces a unique continuous 
linear map $A \hat{\otimes} X \rightarrow X$ mapping $u \otimes x$ to $u x$. If $X$ is a left $A$-module, then the map $u \rightarrow \alpha(u)(\alpha(u) x=u x)$ defines a unital algebra homomorphism $\alpha$ of $A$ into $L(X)$, the algebra of continuous linear maps on $X$. This map is continuous if $L(X)$ is given the topology of simple convergence (strong operator topology). We call $\alpha$ the representation of $A$ associated with the module. Sometimes we shall find it convenient to use the module terminology and notation, while other times it will be more convenient to use the representation point of view.

Right $A$-modules are defined in an analogous fashion. Here, the module operation $(x, u) \rightarrow x u: X \times A \rightarrow X$ corresponds to an antihomomorphism $\alpha: A \rightarrow L(X)$ with $\alpha(u) x=x u$. An $A$-bimodule is an 1.c.s. with left and right $A$-moduler operations which commute with each other $(u(x v)=(u x) v)$.

If $A$ is an algebra, then $A \hat{\otimes} A$ has both an $A$-bimodule structure and its own algebra structure. The bimodule operations we shall use are defined by $u(v \otimes w)=u v \otimes w$ and $(v \otimes w) u=v \otimes w u$. The algebra operation we use on $A \hat{\otimes} A$ will be denoted $(f, g) \rightarrow f * g$ and is defined by $(u \otimes v) *\left(u^{\prime} \otimes v^{\prime}\right)=u u^{\prime} \otimes v^{\prime} v$ (multiplication is reversed in the second factor). Note that, with this notation, the module operations can be written as $u f=(u \otimes 1) * f, f u=(1 \otimes u) * f$.

We shall use the notation $\delta u$ for the element $u \otimes 1-1 \otimes u \in A \otimes A$ determined by $u \in A$.

DEFINITION 1.1. Let $A$ be an algebra and $F_{n} \rightarrow A$ a unital homomorphism with $z_{1}, \ldots, z_{n}$ the images of the generators. We shall say $F_{n} \rightarrow A$ is a localization if there exist continuous linear maps $\Delta_{i}: A \rightarrow A \hat{\otimes} A$ (for $i=1, \ldots, n)$ satisfying

(a) $\Delta_{i} z_{j}=\delta_{i j}(1 \otimes 1), i=1, \ldots, n$;

(b) $\Delta_{i}(u v)=u \Delta_{i}(v)+\Delta_{i}(u) v$ for $u, v \in A, i=1, \ldots, n$;

(c) $\delta u=\left(\Delta_{1} u\right) * \delta z_{1}+\cdots+\left(\Delta_{n} u\right) * \delta z_{n}$.

The above conditions should remind one of similar conditions satisfied by the partial differentiation operators $\partial / \partial z_{i}$ for holomorphic functions of several variables $z_{1}, \ldots, z_{n}$. However, this analogy cannot be carried too far. In fact, the operators $\Delta_{i}$ are more closely related to difference quotients than actual derivatives.

As an example, we consider the algebra $\mathcal{O}(U)$ of holomorphic functions on a domain $U \subset C$. The natural embedding $P \rightarrow \mathcal{O}(U)$ is a localization of $F_{1}=P$. The algebra $\mathcal{O}(U) \hat{\otimes} \mathcal{O}(U)$ may be identified with the algebra $\mathcal{O}(U \times U)$ of holomorphic functions of $(z, w) \in U \times U$ (cf. [12, Theorem 51.6]). The bimodule operations $(u, f) \rightarrow u f$ and $(f, u) \rightarrow f u$ are then given by $(u f)(z, w)=u(z) f(z, w)$ and $(f u)(z, w)=u(w) f(z, w)$. Also, for $u \in \mathcal{O}(U)$ the element $\delta u$ is given by $\delta u(z, w)=u(z)-u(w)$. Thus, it is apparent that (a), (b), and (c) of Definition 1.1 will be satisfied if we define $\Delta: \mathcal{O}(U)$ $\rightarrow \mathcal{O}(U \times U)$ by 


$$
\Delta u(z, w)=(z-w)^{-1}(f(z)-f(w)) .
$$

Of course, it is crucial that the derivative $f^{\prime}(z)$ provides a holomorphic extension of this difference quotient to all of $U \times U$. Thus, the existence of $\Delta$ is related to the existence of the derivative in $\mathcal{O}(U)$, but $\Delta$ itself is the difference quotient.

Throughout the remainder of this section we shall assume that $F_{n} \rightarrow A$ is a localization of $F_{n}$ with generators $z_{1}, \ldots, z_{n}$.

If $\alpha$ is a representation of $A$ on $X$, then we shall write $u(\alpha)$, rather than $\alpha(u)$, for the element in $L(X)$ corresponding to $u \in A$. The reasons for this will soon become apparent. If $\alpha$ and $\beta$ are representations on $X$ and $Y$ and if $c \in L(Y, X)$, then the trilinear map $(u, v, x) \rightarrow u(\alpha) c u(\beta) x: A \times A$ $\times X \rightarrow Y$ is separately continuous and, hence, induces a continuous linear map $\mathscr{N}_{c}: A \hat{\otimes} A \hat{\otimes} X \rightarrow Y$. It follows that each $f \in A \hat{\otimes} A$ defines a linear map $c \rightarrow f(\alpha, \beta)[c]: L(X, Y) \rightarrow L(X, Y)$ by $f(\alpha, \beta)[c](x)=\mathscr{N}_{c}(f \otimes x)$. If $f=u \otimes v$ then $f(\alpha, \beta)[c]$ is just $u(\alpha) c v(\beta)$. If we denote $z_{i}(\alpha)$ by $a_{i}$ and $z_{i}(\beta)$ by $b_{i}$, then $\delta z_{i}(\alpha, \beta)[c]=a_{i} c-c b_{i}$. Condition (c) of Definition 1.1 then implies that

$$
u(\alpha) c-c u(\beta)=\Delta_{1} u(\alpha, \beta)\left[a_{1} c-c b_{1}\right]+\cdots+\Delta_{n} u(\alpha, \beta)\left[a_{n} c-c b_{n}\right]
$$

for all $u \in A$.

Equation (1.1) has several consequences. In the case $X=Y$ and $c=1 \in L(X)$, we conclude that two representations $\alpha$ and $\beta$ of $A$ on $X$ agree if they agree on the generators $\left(a_{i}=b_{i}\right)$. Hence, a representation $\alpha$ of $A$ is uniquely determined by the tuple of operators $\left(a_{1}, \ldots, a_{n}\right)\left(a_{i}=z_{i}(\alpha)\right)$; for this reason, we will henceforth specify a representation by specifying the pair $(X, \alpha)$ with $\alpha=\left(a_{1}, \ldots, a_{n}\right)$. The operators $a_{1}, \ldots, a_{n}$ will be called the generators of the representation. The image of $u \in A$ under the representation will be denoted $u(\alpha)$.

A homomorphism $c: Y \rightarrow X$ between two representations $(X, \alpha)$ and $(Y, \beta)$ is an element $c \in L(Y, X)$ such that $u(\alpha) c=c u(\beta)$ for all $u \in A$ (a left $A$-module homomorphism). It follows from (1.1) that $c$ is a homomorphism if and only if $a_{i} c=c b_{i}$ for $i=1, \ldots, n$, where $a_{1}, \ldots, a_{n}$ and $b_{1}, \ldots, b_{n}$ are the generators. For a single representation generated by $\alpha=\left(a_{1}, \ldots\right.$, $a_{n}$ ), this implies that each $u(\alpha)$ commutes with every operator in $L(X)$ that commutes with each $a_{i}$.

Now every tuple of operators $\alpha=\left(a_{1}, \ldots, a_{n}\right)$ on a complete 1.c.s. $X$ determines a unique representation of $F_{n}$ in which the generators are mapped to $a_{1}, \ldots, a_{n}$. In particular, the elements $z_{1}, \ldots, z_{n} \in A$ determine the canonical embedding $F_{n} \rightarrow A$ of $F_{n}$ in $A$. With this in mind, the preceding discussion may be summarized as follows:

Proposition 1.1. Let $F_{n} \rightarrow A$ be a localization of $F_{n}$. Then 
(a) if $(X, \alpha)$ is a representation of $F_{n}$, there is at most one extension to a representation $u \rightarrow u(\alpha)$ of $A$; if such an extension exists, each $u(\alpha)$ commutes with every $c \in L(X)$ that commutes with the generators $a_{1}, \ldots, a_{n}$;

(b) given two A-modules $(X, \alpha)$ and $(Y, \beta)$ and $c \in L(Y, X), c$ is an A-module homomorphism if and only if it is an $F_{n}$-module homomorphism.

The properties expressed in Proposition 1.1 are not unique to localizations of $F_{n}$. In fact, we could draw the same conclusions if we simply assumed that for each $u \in A$ there were elements $f_{1}, \ldots, f_{n} \in A \hat{\otimes} A$ (not necessarily unique) for which

$$
\delta u=f_{1} * \delta z_{1}+\cdots+f_{n} * \delta z_{n} .
$$

This is a property possessed by all factor algebras of $F_{n}$ or any of its localizations. Such an algebra would be called a pseudo-quotient of $F_{n}$ in the terminology of [11]. The fact that, for a localization, the $f_{i}$ 's are given by continuous linear maps $\Delta_{i}: A \rightarrow A \hat{\otimes} A$ with properties (a) and (b) of Definition 1.1 yields an additional property that is quite strong.

Suppose that $(X, \alpha)$ and $(Y, \beta)$ are representations of $A$. For each $n$-tuple $\left(c_{1}, \ldots, c_{n}\right)$ in $L(Y, X)$ we consider the representation of $F_{n}$ on $X \oplus Y$ generated by the tuple $\gamma=\left(g_{1}, \ldots, g_{n}\right)$, where $g_{i}(x, y)=\left(a_{i} x+c_{i} y, b_{i} y\right)$. In other words, $g_{i}$ is represented by the matrix

$$
\left(\begin{array}{cc}
a_{i} & c_{i} \\
0 & b_{i}
\end{array}\right)
$$

The maps $\Delta_{i}$ give us an explicit way of extending this representation to a representation of $A$ on $X \oplus Y$. We simply let $u(\gamma)$, for $u \in A$, be the operator whose matrix is

$$
\left(\begin{array}{cc}
u(\alpha) & \sum_{i} \Delta_{i} u(\alpha, \beta)\left[c_{i}\right] \\
0 & u(\beta)
\end{array}\right) .
$$

Then $u(\gamma) v(\gamma)$, for $u, v \in A$, is represented by

$$
\left(\begin{array}{cc}
u(\alpha) v(\alpha) & u(\alpha) \sum_{i} \Delta_{i} v(\alpha, \beta)\left[c_{i}\right]+\sum_{i} \Delta_{i} u(\alpha, \beta)\left[c_{i}\right] v(\beta) \\
0 & u(\beta) v(\beta)
\end{array}\right) .
$$

But it follows from (b) of Definition 1.1 that

$$
u(\alpha) \sum_{i} \Delta_{i} v(\alpha, \beta)\left[c_{i}\right]+\sum_{i} \Delta_{i} u(\alpha, \beta)\left[c_{i}\right] v(\beta)=\sum_{i} \Delta_{i} u v(\alpha, \beta)\left[c_{i}\right] .
$$

Hence, $u(\gamma) v(\gamma)=u v(\gamma)$ and $u \rightarrow u(\gamma)$ is a homomorphism. The continuity follows from the continuity of the $\Delta_{i}$ 's. Hence, $u \rightarrow u(\gamma)$ is a representation of $A$. That it extends the given representation of $F_{n}$ follows from (a) of Definition 1.1. 
The representation of $F_{n}$ on $X \oplus Y$ defined above has the property that the injection $X \rightarrow X \oplus Y$ and projection $X \oplus Y \rightarrow Y$ are homomorphisms. That is, $(X, \alpha)$ is a submodule of $(X \oplus Y, \gamma)$ and $(Y, \beta)$ is a factor module. In general, if $X$ and $Y$ are modules over an algebra $A$, then an $A$-module structure on $X \oplus Y$ for which the canonical image of $X$ in $X \oplus Y$ is a submodule and $Y$ is the corresponding factor module is called an $A$-module extension of $Y$ by $X$. Thus, we have proved that

Proposition 1.2. If $F_{n} \rightarrow A$ is a localization and $X$ and $Y$ are $A$-modules, every $F_{n}$-module extension of $Y$ by $X$ is also an A-module extension.

We have already pointed out that the algebras $\mathcal{O}(U)$ are localizations of $F_{1}=P$. Are there others? A moment's reflection should convince the reader that the algebra $C^{\infty}(I)(I$ an open interval on the line) is a localization with generator the identity function. Here again, the difference quotient provides the map $\Delta$. It turns out that the algebra $\mathscr{E}^{\prime}(R)$ of compactly supported distributions is a localization with the derivative of the Dirac delta as generator (cf. [11, §3]). Algebras of rational functions are also localizations (cf. [11, §3]).

Suppose $A$ is any localization of $P$ and let $\Omega$ be the set of those $\lambda \in C$ which generate one-dimensional representations $u \rightarrow u(\lambda)$ of $A$; that is, $\lambda \in \Omega$ if $\lambda$ is the image of the generator $z$ of $A$ under some continuous complex homomorphism of $A$. Then each $u \in A$ determines a function $\lambda \rightarrow u(\lambda)$ on $\Omega$ and each $f \in A \hat{\otimes} A$ determines a function $(\lambda, \omega) \rightarrow f(\lambda, \omega)$ on $\Omega \times \Omega$. Let $\hat{A}$ denote the resulting algebra of functions on $\Omega$. Note that, for $f \in A \hat{\otimes} A, f(\lambda, \omega)$ is in $\hat{A}$ as a function of $\lambda(\omega)$ for each fixed $\omega \in \Omega(\lambda \in \Omega)$. Furthermore, we have

$$
u(\lambda)-u(\omega)=(\lambda-\omega) \Delta u(\lambda, \omega)
$$

from (1.1). It follows that if the elements of $\hat{A}$ are bounded on a subset $S \subset \Omega$, they are also continuous on $S$ and differentiable in the sense that $(\lambda-\omega)^{-1}(u(\lambda)-u(\omega))$ converges as $\lambda \rightarrow \omega$ within $S$. In particular, each $\lambda \rightarrow u(\lambda)$ must be holomorphic on any open set $S$ with this property. One way of insuring that there are large subsets of $\Omega$ on which functions in $\hat{A}$ are bounded is to demand that $A$ be an $F$-algebra (cf. [4]). This insures that $\Omega$ is the union of a sequence of compact sets on which each $\lambda \rightarrow u(\lambda)$ is bounded.

The mapping $A \rightarrow \hat{A}$ defined above need not be one to one for a localization $A$ of $P$. An example is the algebra of all convergent power series. Here $\Omega$ is the single point $\{0\}$.

There are probably too many pathological examples of localizations of $P$ for a complete characterization to be practical. However, as we shall see in $\S 3$, by insisting that $A$ be an $F$-algebra and that $\Delta$ satisfy a certain 
regularity condition relative to a family of seminorms, we eliminate all examples except the algebras $\mathcal{O}(U)$.

2. Extending representations of $F_{n}$. Given a unital algebra homomorphism $F_{n} \rightarrow A$ and a representation $F_{n} \rightarrow L(X)$ of $F_{n}$, we consider the problem of extending the representation to a representation $A \rightarrow L(A)$ of $A$ for which the diagram

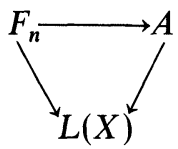

commutes. Since the map $F_{n} \rightarrow A$ is determined by specifying an $n$-tuple $\left(z_{1}, \ldots, z_{n}\right)$ in $A$ and $F_{n} \rightarrow L(X)$ is determined by specifying an $n$-tuple $\left(a_{1}, \ldots, a_{n}\right)$ in $L(X)$, the problem is to find a representation $A \rightarrow L(X)$ which sends $z_{i}$ to $a_{i}$ for $i=1, \ldots, n$. We shall develop a sufficient condition that this be possible and then show that it is both necessary and sufficient if $A$ is a localization of $F_{n}$.

It will be convenient in this section to use module notation and terminology. We consider $F_{n} \otimes F_{n}$ to be an algebra under the operation $(u \otimes v) *\left(u^{\prime} \otimes v^{\prime}\right)=u u^{\prime} \otimes v^{\prime} v$. If $M$ is a right $F_{n}$-module and $X$ a left $F_{n}$-module, then we consider $M \hat{\otimes} X$ to be a right $F_{n} \otimes F_{n}$-module with operation determined by $(m \otimes x) *(u \otimes v)=m u \otimes v x$. With $\delta u=u \otimes 1$ $-1 \otimes u$ for $u \in F_{n}$, we define a map $\theta: \oplus^{n}(M \otimes X) \rightarrow M \otimes X$ by

$$
\theta\left(f_{1} \oplus \cdots \oplus f_{n}\right)=f_{1} * \delta z_{1}+\cdots+f_{n} * \delta z_{n},
$$

where $z_{1}, \ldots, z_{n}$ are the generators of $F_{n}$.

We shall be dealing with right modules with a distinguished element $e \in M$. Such a module will be called an augmented right $F_{n}$-module. For an augmented right $F_{n}$-module $M$ and a left $F_{n}$-module $X$ we may define a map $\tilde{e}: X \rightarrow M \hat{\otimes} X$ by $\tilde{e}(x)=e \otimes x$ and a map $\mathcal{N}: \oplus^{n}(M \hat{\otimes} X) \oplus X$ $\rightarrow M \hat{\otimes} X$ by

$$
\mathcal{N}\left(f_{1} \oplus \cdots \oplus f_{n} \oplus x\right)=\theta\left(f_{1} \oplus \cdots \oplus f_{n}\right)+\tilde{e}(x) .
$$

Definition 2.1. We shall say that the augmented right $F_{n}$-module $M$ dominates the left $F_{n}$-module $X$ and write $M \gg X$ provided the map $\mathcal{N}$ of (2.2) is invertible.

The significance of the condition $M \gg X$ is as follows: If $\mathcal{N}$ is invertible then there is a surjective map $\varphi=\pi \circ \mathcal{N}^{-1}: M \hat{\otimes} X \rightarrow X$, where $\pi$ projects $\oplus^{n}(M \hat{\otimes} X) \oplus X$ onto $X$. The kernel of $\varphi$ is precisely the image of $\theta: \oplus^{n}(M \hat{\otimes} X) \rightarrow M \hat{\otimes} X$. In fact, the existence of $\mathscr{N}^{-1}$ is equivalent to the existence of a map $\varphi: M \hat{\otimes} X \rightarrow X$ for which the sequence

$$
0 \rightarrow \bigoplus^{n}(M \hat{\otimes} X) \stackrel{\oplus}{\rightarrow} M \hat{\otimes} X \stackrel{\leftrightarrow}{ } X \rightarrow 0
$$


is exact and split in the sense that $\theta$ has a left inverse and $\varphi$ a right inverse.

Now if $F_{n} \rightarrow B$ is a unital algebra homomorphism, then we may consider $B$ to be an augmented right $F_{n}$-module where the distinguished element $e$ is the identity 1 . However, $B \hat{\otimes} X$, for any $X$, is a left $B$-module under the operation determined by $u(v \otimes x)=u v \otimes x$. If we choose $M=B$ in (2.3), then it is easy to see that $\theta$ is a left $B$-module homomorphism. It follows that $\varphi$ identifies $X$ with a factor module of $B \hat{\otimes} X$. The space $X$ is already a left $F_{n}$-module, but it is easy to see that $\varphi$ is an $F_{n}$ module homomorphism. Hence, the $B$-module structure induced on $X$ by $\varphi$ extends the given $F_{n}$-module structure. Thus, we have

Proposition 2.1. If $B \gg X$ then $X$ has a $B$-module structure which extends its $F_{n}$-module structure.

Now suppose $F_{n} \rightarrow A$ is a localization and $X$ is a left $A$-module. We define a map $\lambda: A \hat{\otimes} X \rightarrow \oplus^{n}(A \hat{\oplus} X) \oplus X$ by

$$
\lambda(u \otimes x)=\left(\Delta_{1} u\right) x \oplus \cdots \oplus\left(\Delta_{n} u\right) x \oplus u x,
$$

where $\left(\Delta_{i} u\right) x$ refers to the image of $\Delta_{i} u \in A \hat{\otimes} A$ under the map $v \otimes w$ $\rightarrow v \otimes w x: A \hat{\otimes} A \rightarrow A \hat{\otimes} X$. Note that

$$
\begin{aligned}
\mathscr{N} \circ \lambda(u \otimes x) & =\left[\left(\Delta_{1} u\right) * \delta z_{1}+\cdots+\left(\Delta_{u} u\right) * \delta z_{n}\right] x+1 \otimes u x \\
& =(u \otimes 1-1 \otimes u) x+1 \otimes u x=u \otimes x,
\end{aligned}
$$

and

$$
\begin{aligned}
\lambda \circ \mathscr{N}\left[\left(u_{1} \otimes\right.\right. & \left.\left.x_{1}\right) \oplus \cdots \oplus\left(u_{n} \otimes x_{n}\right) \oplus x\right] \\
& =\lambda\left[\sum_{i}\left(u_{i} z_{i} \otimes x_{i}-u_{i} \otimes z_{i} x_{i}\right)+1 \otimes x\right] \\
& =\sum_{j}\left[\sum_{i} \oplus\left(\left[\Delta_{j} u_{i}\right] z_{i} x_{i}+u_{i}\left[\Delta_{j} z_{i}\right] x_{i}-\left[\Delta_{j} u_{i}\right] z_{i} x\right)\right] \oplus x \\
& =\left(\sum_{i} \oplus u_{i} \otimes x_{i}\right) \oplus x .
\end{aligned}
$$

Hence, $\lambda=\mathscr{N}^{-1}$ and we have proved

Proposition 2.2. If $F_{n} \rightarrow A$ is a localization, then a left $F_{n}$-module is also a left $A$-module if and only if $A \gg X$. In this case, the unique A-module structure on $X$ (extending the given $F_{n}$-module structure) is explicitly determined by the condition that the map $\varphi$ of (2.3) be an A-module homomorphism.

In the next section we will need to consider the relation $B \gg C$ for algebras $B$ and $C$ which are completions of $A$ in stronger topologies. More 
generally, suppose $F_{n} \rightarrow A$ is a localization and $\alpha: A \rightarrow B, \beta: A \rightarrow C$ are unital algebra homomorphisms with $\alpha$ having dense range. As before, we consider $B$ to be an augmented right $F_{n}$-module and $C$ a left $F_{n}$-module. If $B \gg C$ then the map $\varphi: B \hat{\otimes} C \rightarrow C$ of (2.3) induces an $F_{n}$-module homomorphism $\gamma: B \rightarrow C$, where $\gamma(u)=\varphi(u \otimes 1)$. However, $B$ and $C$ are also left $A$-modules via the maps $\alpha$ and $\beta$ and so, by Proposition 1.1, $\gamma$ is also an $A$-module homomorphism. The fact that $A$ embeds densely in $B$ then implies that $\gamma$ is in fact an algebra homomorphism from $B$ to $C$ for which the diagram

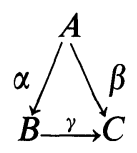

is commutative. Thus, $B \gg C$ implies that $\beta: A \rightarrow C$ factors through $\alpha: A \rightarrow B$. We can conclude even more, however: We note that if $u \in B$ then $u \otimes 1-1 \otimes \gamma(u) \in B \hat{\otimes} C$ is in the kernel of $\varphi: B \hat{\otimes} C \rightarrow C$. Since the map $\theta$ of (2.3) has a left inverse, $\theta^{-1}$, and (2.3) is exact, we may define a $\operatorname{map} \bar{\Delta}: B \rightarrow \bigoplus^{n}(B \hat{\otimes} C)$ by

$$
\bar{\Delta}(u)=\theta^{-1}(u \otimes 1-1 \otimes \gamma(u)) .
$$

If $\Delta: A \rightarrow \bigoplus^{n}(A \hat{\otimes} A)$ is the map whose coordinate maps are our $\Delta_{i}$ 's, then it is easy to see that the diagram

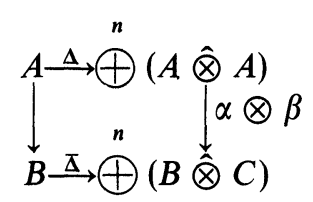

is commutative. Hence, $\bar{\Delta}$ provides an extension of $\Delta$ to a map from $B$ to $\bigoplus^{n}(B \otimes C)$.

Conversely, if $\beta$ factors through $\alpha$ and $\Delta$ extends to a map $\bar{\Delta}: B \rightarrow$ $\bigoplus^{n}(B \hat{\otimes} C)$, then an argument like the one preceding Proposition 2.2 shows how to construct an inverse for $\mathscr{N}$. Hence,

Proposition 2.3. Let $F_{n} \rightarrow A$ be a localization and $\alpha: A \rightarrow B, \beta: A \rightarrow C$ be unital algebra homomorphisms, with $\alpha$ having dense range. Then the relation $B \gg C$ holds if and only if $\beta$ factors through $\alpha$ and $\Delta: A \rightarrow$ $\bigoplus^{n}(A \hat{\otimes} A)$ extends continuously to a map $\bar{\Delta}: B \rightarrow \bigoplus^{n}(B \hat{\otimes} C)$.

The existence of the map $\bar{\Delta}: B \rightarrow \bigoplus^{n}(B \hat{\otimes} C)$ can also be used to construct an inverse for $\mathscr{N}$ for the pair $B, X$, where $X$ is any left $C$-module. Since $B \gg C$ is equivalent to the existence of such a $\bar{\Delta}$, we have

Proposition 2.4. For the localization $F_{n} \rightarrow A$, let $\alpha: A \rightarrow B$ and $\beta: A \rightarrow C$. 
be as in Proposition 2.3. Then $B \gg C$ implies $B \gg X$ for any left $C$-module $X$.

A major reason that the relation " $\gg$ " is useful is that it satisfies a rather strong stability property. Suppose $M$ is an augmented right $F_{n}$-module which happens to be a Banach space, and $X$ is any Banach space. If $\beta=\left(b_{1}, \ldots, b_{n}\right)$ is a tuple of operators on $X$, we denote the left $F_{n}$-module in which the generators of $F_{n}$ act as the operators $b_{1}, \ldots, b_{n}$ by $(X, \beta)$. For this situation, the map $\mathscr{N}$ of (2.2) is a bounded linear map between two Banach spaces. Furthermore, as the $b_{i}$ 's vary (varying the module structure on $X$ ), the map $\mathcal{N}$ varies in a continuous fashion. It follows that if $\mathscr{N}$ is invertible for one tuple $\alpha$ it is invertible for all tuples $\beta$ in a (norm) neighborhood of $\alpha$ and $\mathscr{N}^{-1}$ (and, hence $\varphi$ ) varies continuously with $\beta$. It follows that

Proposition 2.5. If $M$ is an augmented right $F_{n}$-module, $M$ and $X$ Banach spaces, and $(X, \beta)$ is the left $F_{n}$-module determined by a tuple $\beta=\left(b_{1}, \ldots, b_{n}\right)$ of elements of $L(X)$, then the set of $\beta$ for which $M \gg(X, \beta)$ is open in the norm topology of $\bigoplus^{n} L(X)$ and the induced map $\varphi: M \otimes X$ $\rightarrow X$ varies continuously with $\beta$ on this set.

In the particular case where $M$ is a Banach algebra $B$ with an embedding $F_{n} \rightarrow B$, the continuity of $\varphi$ as a function of $\beta$ implies that the $B$-module structure induced on $(X, \beta)$ (as in Proposition 2.1) varies continuously with $\beta$. That is, the representation $u \rightarrow u(\beta): B \rightarrow L(X)$ induced by $\varphi(u(\beta)(x)=\varphi(u \otimes x))$ varies continuously with $\beta$ in the norm topology of $L(B, L(X))$.

In $\$ 7$ we shall continue the study of the relation " $\gg$ " and its applications to the functional calculus problem. There we shall introduce a complementary relation " $\perp$ " which is related to the resolvent set of ordinary spectral theory.

3. Free analytic algebras. The stability property of the relation " $\gg "$ expressed in Proposition 2.5 provides the key to strengthening the concept of localization so as to obtain a class of algebras which are generalizations to the case of several noncommuting variables of the algebras $\mathcal{O}(U)$. An important property of an algebra $\mathcal{O}(U)$ is the following: If $P \rightarrow L(X)$ is a representation of the polynomial algebra on a Banach space $X$ (generated by $a \in L(X)$ ) which extends to a representation of $\mathcal{O}(U)$ (i.e., $\mathrm{Sp}(a) \subset U)$, then all nearby representations of $P$ also extend to $\mathcal{O}(U)$ $(\mathrm{Sp}(b)$ remains in $U$ for all $b$ in some neighborhood of $a)$. It is this property we would like to recapture in several variables.

Definition 3.1. Let $F_{n} \rightarrow A$ be a localization of $F_{n}$ with $\Delta_{1}, \ldots, \Delta_{n}$ the maps of Definition 1.1. We shall say that $A$ is a free analytic algebra on $n$-generators if there is a sequence $\left\{\rho_{k}\right\}$ of submultiplicative seminorms on 
$A$, which defines the topology of $A$ and has the property that for each $k>1$ the maps $\Delta_{i}$ are continuous from $A$ with the $\rho_{k}$ seminorm to $A \hat{\otimes} A$ with the cross seminorm $\rho_{k} \times \rho_{k-1}$.

If $A_{k}$ denotes the algebra obtained from $A$ by factoring out the null ideal of $\rho_{k}$ and completing in the $\rho_{k}$ norm, then the content of Definition 3.1 is that each $\Delta_{i}$ extends continuously to a map from $A_{k}$ to $A_{k} \hat{\otimes} A_{k-1}$. By Proposition 2.3 this is equivalent to the relation $A_{k} \gg A_{k-1}$ for each $k$. Now suppose $u \rightarrow u(\alpha): A \rightarrow L(X)$ is a representation of $A$ (generated by the tuple $\alpha=\left(a_{1}, \ldots, a_{n}\right)$ of elements of $\left.L(X)\right)$ on a Banach space $X$. Since the $\rho_{i}$ 's determine the topology of $A$, there is some $i$ for which the representation factors through the map $A \rightarrow A_{i-1}$ and, hence, determines a representation $A_{i-1} \rightarrow L(X)$. By Proposition 2.4, this implies that $A_{i} \gg(X, \alpha)$. By Proposition 2.5 , the same relation holds for all $\beta=$ $\left(b_{1}, \ldots, b_{n}\right)$ in a neighborhood of $\alpha$ (in the norm topology of $\bigoplus^{n}(L(X))$. By Proposition 2.1, the representation of $F_{n}$ determined by each such $\beta$ extends to a representation of $A_{i}$ and, hence, to a representation $u \rightarrow u(\beta)$ of $A$. From this, and the comment following Proposition 2.5, we conclude

Proposition 3.1. Let $A$ be a free analytic algebra and $X$ a Banach space. Then the set of all $\alpha \in \bigoplus^{n} L(X)$ for which $(X, \alpha)$ is an $A$-module is an open set in the norm topology. Furthermore, for each $\alpha$ in this set there is a neighborhood $V$ of $\alpha$ and an integer $i$ such that the representation $u \rightarrow u(\beta)$ factors through $A \rightarrow A_{i}$ for each $\beta \in V$ and varies continuously with $\beta$ in norm as an element of $L\left(A_{i}, L(X)\right)$.

We recall some notation from $\S 1$. If $u \rightarrow u(\alpha)$ and $u \rightarrow u(\beta)$ are representations of $A$ on $X$ and $Y$ respectively, then for each $c \in L(Y, X)$ there is a map $u \otimes v \rightarrow u(\alpha) c u(\beta): A \hat{\otimes} A \rightarrow L(Y, X)$. We denote the image of $f \in A \hat{\otimes} A$ under this map by $f(\alpha, \beta)[c]$. Then $c \rightarrow f(\alpha, \beta)[c]$ is a linear map from $L(Y, X)$ to itself for each $f \in A \hat{\otimes} A$. Now if $X$ and $Y$ are Banach spaces and $u \rightarrow u(\alpha)$ and $u \rightarrow u(\beta)$ each factor through $A \rightarrow A_{i}$ then the map $c \rightarrow f(\alpha, \beta)[c]$ is a bounded linear map from $L(Y, X)$ to $L(Y, X)$ with norm dominated by the norm of $f$ in the Banach space $A_{i} \hat{\otimes} A_{i}$. In fact, it follows from Proposition 3.1 that the map $c \rightarrow f(\alpha, \beta)[c]$ varies continuously with $(\alpha, \beta)$ and satisfies a uniform bound for $\alpha$ and $\beta$ in sufficiently small neighborhoods $U \subset \oplus^{n} L(X), V \subset \bigoplus^{n} L(Y)$.

If we choose $X=Y$ and $c=1$ in formula (1.1), note we obtain the formula

$$
u(\alpha)-u(\beta)=\Delta_{1} u(\alpha, \beta)\left[a_{1}-b_{1}\right]+\cdots+\Delta_{n} u(\alpha, \beta)\left[a_{n}-b_{n}\right] .
$$

If we set $\beta=\alpha+\lambda \gamma$ for $\gamma=\left(c_{1}, \ldots, c_{n}\right)$ and $\lambda \in \boldsymbol{C}$, this implies

$$
\lambda^{-1}[u(\alpha+\lambda \gamma)-u(\alpha)]=\Delta_{1} u(\alpha, \alpha+\lambda \gamma)\left[c_{1}\right]+\cdots+\Delta_{n} u(\alpha, \alpha+\lambda \gamma)\left[c_{n}\right] .
$$


On taking the limit as $\lambda \rightarrow 0$ we conclude

Proposition 3.2. If $A$ is a free analytic algebra and $X$ a Banach space, then on the set of $\alpha \in \bigoplus^{n} L(X)$ for which $(X, \alpha)$ determines a representation of $A$, each of the functions $u(\alpha)$ for $u \in A$ is an analytic function of $\alpha$ with derivative given by

$$
\left.\frac{d}{d \lambda} u(\alpha+\lambda \gamma)\right|_{\lambda=0}=\Delta_{1} u(\alpha, \alpha)\left[c_{1}\right]+\cdots+\Delta_{n} u(\alpha, \alpha)\left[c_{n}\right]
$$

for $\gamma=\left(c_{1}, \ldots, c_{n}\right)$.

The free analytic algebras on one generator are easily characterized. Recall from $\S 1$ that if $P \rightarrow A$ is a localization of the polynomial algebra, then we denote by $\Omega$ the set of all $\lambda \in C$ for which the one-dimensional representation of $P$ determined by $z \rightarrow \lambda$ extends to a representation (complex homomorphism) $u \rightarrow \hat{u}(\lambda)$ of $A$. If $A$ is a free analytic algebra then it follows from Proposition 3.1 that this set is open and each $\hat{u}$ is a continuous function on $\Omega$. By Proposition 3.2, each $\hat{u}$ is, in fact, analytic. It follows that $u \rightarrow \hat{u}$ is a homomorphism of $A$ into $\mathcal{O}(\Omega)$. Now $A$ is a commutative $F$-algebra (projective limit of a sequence of commutative Banach algebras) and the spectrum of the generator $z \in A$ is exactly $\Omega$. Hence, it follows from the analytic functional calculus for Fréchet algebras (cf. [4]) that there is a homomorphism $\mathcal{O}(\Omega) \rightarrow A$ so that the composition $\mathcal{O}(\Omega) \rightarrow A \rightarrow^{u \rightarrow \hat{u}} \mathcal{O}(\Omega)$ is the identity. Since $A$ is a localization of $P$ and $\mathcal{O}(\Omega)$ is an $A$-module via the map $A \rightarrow \mathcal{O}(\Omega)$ it follows from Proposition 1.1 that the $P$-module homomorphism $\mathcal{O}(\Omega) \rightarrow A$ must also be an $A$-module homomorphism. This implies that $\mathcal{O}(\Omega)$ is isomorphic to an ideal in $A$. Hence, $A=\mathcal{O}(\Omega) \oplus K$ is the direct sum of ideals, where $K=\operatorname{ker}(u \rightarrow \hat{u})$. This is impossible if $K \neq 0$ since $K$ would also be a commutative $F$-algebra with identity and, hence, have at least one nontrivial complex homomorphism. This complex homomorphism would extend to a complex homomorphism of $A$ not determined by a point of $\Omega$. Thus,

Proposition 3.3. If $A$ is a free analytic algebra on one generator, then $A=\mathcal{O}(U)$ for some domain $U \subset C$.

In order to prove the converse, we must show that the map $\Delta: \mathcal{O}(U)$ $\rightarrow \mathcal{O}(U \times U)$ given by $\Delta u(\lambda, \omega)=(\lambda-\omega)^{-1}(u(\lambda)-u(\omega))$ is continuous from the $\rho_{k}$ to the $\rho_{k} \times \rho_{k-1}$ seminorms for some defining sequence of seminorms for the topology of $\mathcal{O}(U)$.

We choose a sequence of compact sets $K_{1}, K_{2}, \cdots \subset U$ with $K_{i-1}$ $\subset \operatorname{int}\left(K_{i}\right)$ and the property that every compact set in $U$ is contained in some $K_{i}$. We then set $\rho_{i}(u)=\sup _{K_{i}}|u(\lambda)|$. The resulting family of semi- 
norms generates the topology of $\mathcal{O}(U)$. Unfortunately, the greatest cross norm $\rho_{i} \times \rho_{i-1}$ on $\mathcal{O}(U \times U)$ is difficult to describe. It is not simply sup norm on $K_{i} \times K_{i-1}$; this is the least cross norm generated by $\rho_{i}$ and $\rho_{i-1}$ (cf. [2], [6]). Hence, we must resort to some trickery.

Given any compact set $L \subset \operatorname{int}\left(K_{i}\right)$, it follows easily from the Cauchy integral theorem that there is an $M$ so that $\sup \left\{|\Delta u(\lambda, \omega)|:(\lambda, \omega) \in K_{i} \times L\right\}$ $\leqq M \rho_{i}(u)$ for every $u \in \mathcal{O}(U)$. Thus, if $B$ is the completion of $\mathcal{O}(U)$ in the topology of uniform convergence on compact sets in $V$ for some open $V \subset K_{i}$ and if $A_{i}$ is the completion of $\mathcal{O}(U)$ in the norm $\rho_{i}$, then $\Delta$ extends continuously to a map from $A_{i}$ to the completion of $A_{i} \otimes B$ in the topology of compact convergence on $K_{i} \times V$. However, this is exactly the topology of bi-equicontinuous convergence for $A_{i} \otimes B$ (cf. [12, 44.1]). Furthermore, $B$ is a nuclear space (cf. $[12, \S 51]$ ) and, hence, the topology of equicontinuous convergence on $A_{i} \otimes B$ agrees with the projective tensor product topology (cf. $[12,50.1])$. It follows that $\Delta$ extends continuously to a map from $A_{i}$ to $A_{i} \hat{\otimes} B$. Now if $V$ is chosen so that $K_{i-1} \subset V \subset K_{i}$, then the restriction map $B \rightarrow A_{i-1}$ is continuous and induces a continuous map $A_{i} \hat{\otimes} B \rightarrow A_{i} \hat{\otimes} A_{i-1}$. Hence, $\Delta$ extends continuously to a map from $A_{i}$ to $A_{i} \hat{\otimes} A_{i-1}$. We conclude that

Proposition 3.4. The algebras $\mathcal{O}(U)$ (with $z$ as generator) are exactly the free analytic algebras on one generator.

Observe that if a localization $A$ of $P$ is a Banach algebra then it is automatically also a free analytic algebra on one generator (with all $\rho_{i}$ 's equal to the norm on $A$ ). Since no algebra $\mathcal{O}(U)$ is a Banach algebra, there are no localizations of $P$ which are Banach algebras. We strongly suspect that the same is true of localizations of $F_{n}$ for arbitrary $n$.

Are there any free analytic algebras on $n$-generators for $n>1$ ? In $[11, \S 6]$, we gave several examples of localizations of $F_{n}$. Some of these are free analytic algebras. Here we shall construct one family of examples (the power series algebras) and in $\$ 5$ give a technique for constructing a wide class of examples by factoring certain ideals out of power series algebras.

We denote the generators of $F_{n}$ by $z_{1}, \ldots, z_{n}$. A given monomial $z_{j_{1}} z_{j_{2}} \ldots z_{j_{k}}$ will be denoted $z_{\sigma}$ where $\sigma=\left(j_{1}, \ldots, j_{k}\right)$. Thus, a typical element of $F_{n}$ is a finite sum $\sum_{\sigma} \lambda_{\sigma} z_{\sigma}$ with each $\lambda_{\sigma} \in \boldsymbol{C}$. If $r=\left(r_{1}, \ldots, r_{n}\right)$ is an $n$-tuple of positive numbers (with $+\infty$ allowed), then $\mathscr{F}_{n}(r)$ will denote the algebra of all infinite series $\sum_{\sigma} \lambda_{\sigma} z_{\sigma}$ for which $\sum_{\sigma}\left|\lambda_{\sigma}\right| t_{\sigma}<\infty$ for every $t=\left(t_{1}, \ldots, t_{n}\right)$ with $0<t_{i}<r_{i}$; here $t_{\sigma}=t_{j_{1}} t_{j_{2}} \ldots t_{j_{k}}$ for $\sigma=\left(j_{1}, \ldots, j_{k}\right)$. Thus, $\mathscr{F}_{n}(r)$ is the completion of $F_{n}$ in the topology determined by the family of seminorms $\left\{\rho_{t}: t=\left(t_{1}, \ldots, t_{n}\right), t_{i}<r_{i}\right\}$, where $\rho_{t}\left(\sum \lambda_{\sigma} z_{\sigma}\right)=\sum\left|\lambda_{\sigma}\right| t_{\sigma}$. We call $\mathscr{F}_{n}(r)$ the free power series algebra of radius 
$r=\left(r_{1}, \ldots, r_{n}\right)$.

We define a map $\Delta_{i}: F_{n} \rightarrow F_{n} \otimes F_{n}$ for $i=1, \ldots, n$ by

$$
\Delta_{i}\left(z_{j_{1}} \ldots z_{j_{k}}\right)=\sum_{l} \delta_{i j_{l}}\left(z_{j_{1}} \ldots z_{j_{l-1}} \otimes z_{j_{l+1}} \ldots z_{j_{k}}\right),
$$

where $\delta_{i j}=0$ for $i \neq j$ and $\delta_{i j}=1$ for $i=j$. It is a simple matter to check that $\Delta_{1}, \ldots, \Delta_{n}$ satisfy the conditions of Definition 1.1. Thus, $F_{n}$ is a localization of $F_{n}$.

If $s$ and $t$ are two positive $n$-tuples and $0<\lambda<1$ with $s_{i} \leqq \lambda t_{i}, i=1, \ldots$, $n$, then for each monomial $z_{j_{1}}, \ldots, z_{j_{k}}=z_{\sigma}$ we have

$$
\begin{aligned}
\rho_{t} \times \rho_{s}\left(\Delta_{i} z_{\sigma}\right) & =\sum_{l} \delta_{i j_{l}} t_{j_{1}} \cdots t_{j_{l-1}} s_{j_{l+1}} \cdots s_{j_{k}} \\
& \leqq \frac{1}{\min \left\{t_{j}\right\}} \sum_{l=1}^{k} t_{j_{1}} \cdots t_{j_{l}} s_{j_{l+1}} \cdots s_{j_{k}} \\
& \leqq \frac{1}{\min \left\{t_{j}\right\}} \sum_{l=1}^{k} \lambda^{k-l} t_{j_{1}} \cdots t_{j_{k}} \\
& \leqq \frac{1}{(1-\lambda) \min \left\{t_{j}\right\}} \rho_{t}\left(z_{\sigma}\right) .
\end{aligned}
$$

Hence, the map $\Delta_{i}$ is continuous from $F_{n}$ with the $\rho_{t}$ norm to $F_{n} \otimes F_{n}$ with the $\rho_{t} \times \rho_{s}$ norm. It follows that for any radius $r=\left(r_{1}, \ldots, r_{n}\right)$ we can choose an increasing sequence of norms $\rho_{t}$ which define the topology of $\mathscr{F}_{n}(r)$ and satisfy the condition of Definition 3.1. Hence,

Proposition 3.5. Each of the power series algebras $\mathscr{F}_{n}(r)$ is a free analytic algebra on n-generators.

4. Taylor's formula and power series expansions. Let $F_{n} \rightarrow A$ be a localization. In this section, we shall construct analogues of higher derivatives for $A$ by iterating the map $\Delta: A \rightarrow \bigoplus^{n}(A \hat{\otimes} A)$ determined by the $\Delta_{i}$ 's.

We define a map $\theta: \oplus^{n}(A \hat{\otimes} A) \rightarrow A \hat{\otimes} A$ by $\theta\left(f_{1} \oplus \cdots \oplus f_{n}\right)=f_{1} * \delta z_{1}$ $+\cdots+f_{n} * \delta z_{n}$ (this is the map $\theta$ of (2.3) in the case $M=X=A$ ). Note that the content of (c) of Definition 1.1 is that

$$
\delta=\theta \circ \Delta, \quad \text { where } \delta u=u \otimes 1-1 \otimes u .
$$

If $\pi: A \otimes A \rightarrow A$ is the multiplication map, then we may construct a map $(1 \otimes \pi) \circ(\Delta \otimes 1): A \hat{\otimes} A \rightarrow \bigoplus(A \hat{\otimes} A)$ by applying $\Delta \otimes 1: A \hat{\otimes} A$ $\rightarrow\left(\oplus^{n}(A \hat{\otimes} A)\right) \hat{\otimes} A=\bigoplus^{n}(A \hat{\otimes} A \hat{\otimes} A)$ and then applying $1 \otimes \pi$ : $A \hat{\otimes}(A \hat{\otimes} A) \rightarrow A \hat{\otimes} A$ in each term. The content of (a) and (b) of Definition 1.1 is that

$$
(1 \otimes \pi) \circ(\Delta \otimes 1) \circ \theta=1,
$$


in fact, if we apply $\theta$ to an element $u \otimes v$ in the ith term of $\bigoplus^{n}(A \hat{\otimes} A)$ we obtain $u z_{i} \otimes v-u \otimes z_{i} v$. Applying $\Delta \otimes 1$ yields in the $j$ th coordinate

$$
\begin{aligned}
& \left(\Delta_{j} u\right) z_{i} \otimes v+u\left(\Delta_{j} z_{i}\right) \otimes v-\Delta_{j} u \otimes z_{i} v \quad \text { (by (b) of Definition 1.1) } \\
& =\Delta_{j} u z_{i} \otimes v+\delta_{i j}(u \otimes 1 \otimes v)-\Delta_{j} u \otimes z_{i} v \quad \text { (by (a) of Definition 1.1). }
\end{aligned}
$$

If we apply $1 \otimes \pi$ to this, the first and third terms cancel and the middle term becomes $\delta_{i j}(u \otimes v)$. This establishes (4.2). Since $\theta$ has a left inverse it is one to one, as are the maps $1 \otimes \theta$ and $\theta \otimes 1$. We shall use this fact to prove that if we iterate $\Delta$ by applying it again on either side we obtain the same map, i.e., that $(1 \otimes \Delta) \circ \Delta=(\Delta \otimes 1) \circ \Delta$.

It will be convenient to choose a basis (over the algebra $A \hat{\otimes} A$ ) for $\bigoplus^{n}(A \hat{\otimes} A)$. Thus, we denote by $e_{i}$ the element of $\bigoplus^{n}(A \otimes A)$ which is $1 \otimes 1$ in the $i$ th coordinate and write $\bigoplus^{n}(A \hat{\otimes} A)=\bigoplus_{i} A e_{i} A$. In general, by $A e_{j_{1}} A \ldots A e_{j_{k}} A$ we shall mean a specific copy of the $k$-fold tensor product $A \hat{\otimes} A \ldots A \hat{\otimes} A$. The maps $(1 \otimes \Delta)$ and $(\Delta \otimes 1)$ applied coordinatewise to $\bigoplus_{i} A e_{i} A$ yield elements of $\bigoplus_{i, j} A e_{i} A e_{j} A$. Hence, $(1 \otimes \Delta) \circ \Delta$ and $(\Delta \otimes 1) \circ \Delta$ are maps from $A$ to $\bigoplus_{i, j} A e_{i} A e_{j} A$.

Note that by our comments above regarding $\theta$, we have $(1 \otimes \theta) \circ(\theta \otimes 1)$ $=(\theta \otimes 1) \circ(1 \otimes \theta): \bigoplus_{i, j} A e_{i} A e_{j} A \rightarrow A \hat{\otimes} A$ is one to one. Thus, in order to prove that $(1 \otimes \Delta) \circ \Delta=(\Delta \otimes 1) \circ \Delta$, it suffices to prove that

$$
(1 \otimes \theta) \circ(\theta \otimes 1) \circ(\Delta \otimes 1) \circ \Delta=(\theta \otimes 1) \circ(1 \otimes \theta) \circ(1 \otimes \Delta) \circ \Delta .
$$

Note that $(\theta \otimes 1) \circ(\Delta \otimes 1)=\delta \otimes 1$ and $(1 \otimes \theta) \circ(1 \otimes \Delta)=1 \otimes \delta$ by (4.1). Hence, we must prove that

$$
[(1 \otimes \theta) \circ(\delta \otimes 1)-(\theta \otimes 1) \circ(1 \otimes \delta)] \circ \Delta \text { is zero. }
$$

If we apply $(1 \otimes \theta) \circ(\delta \otimes 1)-(\theta \otimes 1) \circ(1 \otimes \delta)$ to an element of $\bigoplus_{i} A e_{i} A$ of the form $u e_{i} v$ we obtain

$$
\begin{aligned}
\left(u \otimes z_{i} \otimes v-u \otimes 1 \otimes z_{i} v-1 \otimes u z_{i} \otimes v+1 \otimes u \otimes z_{i} v\right) \\
-\left(u z_{i} \otimes v \otimes 1-u \otimes z_{i} v \otimes 1-u z_{i} \otimes 1 \otimes v+u \otimes z_{i} \otimes v\right) ;
\end{aligned}
$$

on regrouping, this becomes

$$
\begin{aligned}
&-\left(u z_{i} \otimes v-u \otimes z_{i} v\right) \otimes 1-1 \otimes\left(u z_{i} \otimes v-u \otimes z_{i} v\right) \\
&+u z_{i} \otimes 1 \otimes v-u \otimes 1 \otimes z_{i} v .
\end{aligned}
$$

The first and second terms are $-\theta\left(u e_{i} v\right) \otimes 1$ and $-1 \otimes \theta\left(u e_{i} v\right)$, while the third term is $T_{23}\left(\theta\left(u e_{i} v\right) \otimes 1\right)$, where $T_{23}$ is the map $T_{23}: A \hat{\otimes} A \hat{\otimes} A$ $\rightarrow A \hat{\otimes} A \hat{\otimes} A$ which transposes second and third factors. It follows from (4.1) that the expression (4.4) applied to an element $u \in A$ yields 


$$
\begin{aligned}
-\theta \circ \Delta(u) \otimes 1- & 1 \otimes \theta \circ \Delta(u)+T_{23}[\theta \circ \Delta(u) \otimes 1] \\
= & 1 \otimes u \otimes 1-u \otimes 1 \otimes 1+1 \otimes 1 \otimes u \\
& -1 \otimes u \otimes 1+u \otimes 1-1 \otimes 1 \otimes u \\
= & 0 .
\end{aligned}
$$

This completes the proof that

Proposition 4.1. The maps $(1 \otimes \Delta) \circ \Delta$ and $(\Delta \otimes 1) \circ \Delta$ from $A$ to $\sum_{i, j} A e_{i} A e_{j} A$ are identical.

We denote the map $(\Delta \otimes 1) \circ \Delta$ by $\Delta^{2}$. Note that it also follows from Proposition 4.1 that if we define $\Delta^{k}$ inductively by $\Delta^{k}=(\Delta \otimes 1 \otimes \cdots \otimes 1)$ $\Delta^{k-1}$, then

$$
(1 \otimes \cdots \otimes \Delta \otimes \cdots \otimes 1) \circ \Delta^{k-1}=\Delta^{k}
$$

where $\Delta$ can be placed in any slot in $(1 \otimes \cdots \otimes \Delta \otimes \cdots \otimes 1)$. If we set $\theta^{i}=1 \otimes \cdots \otimes \theta \otimes \cdots \otimes 1(\theta$ in the $i$ th place $)$ and $\delta^{i}=1 \otimes \cdots \otimes \delta \otimes \cdots$ $\otimes 1$ ( $\delta$ in the $i$ th place) then equation (4.1) implies that

$$
\delta^{i} \Delta^{k-1}=\theta^{i} \circ \Delta^{k}
$$

Given $k+1$ representations $\left(X, \alpha^{0}\right), \ldots,\left(X, \alpha^{k}\right)$ of $A$ on $X$ and $k$ $n$-tuples $\gamma^{1}, \ldots, \gamma^{k}$ with each $\gamma^{j}=\left(c_{1}^{j}, \ldots, c_{n}^{j}\right)$ a tuple of elements of $L(X)$, we can define, for each $f \in \sum_{j_{1} \cdots j_{k}} A e_{j_{1}} A \cdots A e_{j_{k}} A$, an element $f\left(\alpha^{0}, \ldots, \alpha^{k}\right)\left[\gamma^{1}, \ldots, \gamma^{n}\right] \in L(X)$ by evaluating the factors of $A e_{j_{1}} A \cdots$ $A e_{j_{k}} A$ at the representations $\alpha_{0}, \ldots, \alpha_{k}$ and replacing each $e_{j_{i}}$ by $c_{j_{i}}^{i}$. In other words, if $f=u_{0} e_{j_{1}} u_{1} \cdots u_{k-1} e_{j_{k}} u_{k}$ then

$$
f\left(\alpha^{0}, \ldots, \alpha^{k}\right)\left[\gamma_{1}, \ldots, \gamma_{k}\right]=u_{0}\left(\alpha_{0}\right) c_{j_{1}}^{1} u_{1}\left(\alpha_{1}\right) \ldots u_{k-1}\left(\alpha_{n-1}\right) c_{j_{k}}^{k} u_{k}\left(\alpha_{k}\right) .
$$

If we use this notation, then (4.5) implies that

$\Delta^{k} u\left(\alpha_{0}, \ldots, \alpha_{i-1}, \alpha, \alpha_{i+1}, \ldots, \alpha_{k}\right)\left[\gamma_{1}, \ldots, \gamma_{k}\right]$

$$
\begin{gathered}
-\Delta^{k} u\left(\alpha_{0}, \ldots, \alpha_{i-1}, \beta, \alpha_{i+1}, \ldots, \alpha_{k}\right)\left[\gamma_{1}, \ldots, \gamma_{2}\right] \\
=\Delta^{k+1}\left(\alpha_{0}, \ldots, \alpha_{i-1}, \alpha, \beta, \alpha_{i+1}, \ldots, \alpha_{k}\right)\left[\gamma_{1}, \ldots, \gamma_{i-1}, \alpha-\beta, \gamma_{i}, \ldots, \gamma_{k}\right] .
\end{gathered}
$$

If we begin with an arbitrary pair of representations $\alpha$ and $\beta$ and iterate (4.6) we obtain

$$
\begin{aligned}
u(\alpha)= & u(\beta)+\Delta u(\alpha, \beta)[\alpha-\beta] \\
= & u(\beta)+\Delta u(\beta, \beta)[\alpha-\beta]+\Delta^{2}(\alpha, \beta, \beta)[\alpha-\beta, \alpha-\beta] \\
= & u(\beta)+\Delta u(\beta, \beta)[\alpha-\beta]+\Delta^{2} u(\beta, \beta, \beta)[\alpha-\beta, \alpha-\beta] \\
& +\Delta^{3} u(\alpha, \beta, \beta, \beta)[\alpha-\beta, \alpha-\beta, \alpha-\beta]
\end{aligned}
$$


and so on. Thus,

Proposition 4.2. Let $A$ be a localization of $F_{n}$ and $(\alpha, X)$ and $(\beta, X)$ two representations of $A$ on $X$. Then

$$
\begin{aligned}
u(\alpha)=u(\beta) & +\Delta u(\beta, \beta)[\alpha-\beta]+\cdots \\
& +\Delta^{k} u(\beta, \ldots, \beta)[\alpha-\beta, \ldots, \alpha-\beta] \\
& +\Delta^{k+1}(\alpha, \beta, \ldots, \beta)[\alpha-\beta, \ldots, \alpha-\beta]
\end{aligned}
$$

for each $u \in A$.

Formula (4.7) is our analogue of Taylor's formula. The $k$-linear transformation $\Delta^{k} u(\beta, \ldots, \beta): X^{k}\left(\oplus^{n} L(X)\right) \rightarrow L(X)$ plays the role of the $k$ th coefficient. Note that only for the last term of (4.7) does the coefficient depend on $\alpha$. Note also that if $\beta$ happens to be a tuple of scalars (multiples of the identity) then the coefficients $\Delta^{k} u(\beta, \ldots, \beta)$ must be scalar multilinear forms. This follows from the fact that, for $v \in A, v(\beta)$ commutes with all operators that commute with each entry in the tuple $\beta$ (Proposition 1.1). Thus, in this case, (4.7) represents an honest polynomial in $a_{1}-b_{1}, \ldots, a_{n}-b_{n}$ together with a remainder term.

If $A$ is a free analytic algebra, then for $\alpha$ near $\beta$ we can obtain bounds on the sizes of the coefficients in (4.7) when $X$ is a Banach space and conclude that the resulting power series converges. In fact, if $u \rightarrow u(\beta)$ factors through $A \rightarrow A_{i-1}$, then for $\alpha$ in some neighborhood $V$ of $\beta$ we have $u \rightarrow u(\alpha)$ factors through $A \rightarrow A_{i}$ and satisfies a uniform bound $\|u(\alpha)\| \leqq M \rho_{i}(u)$. Furthermore, the map $\Delta$ extends to a bounded linear map $\Delta: A_{i} \rightarrow \bigoplus^{n}\left(A_{i} \otimes A_{i-1}\right)$. If the norm of this map is $N$ then we conclude that $\left\|\Delta^{k}\right\|=N^{k}$ and

$$
\left\|\Delta^{k} u(\beta, \ldots, \beta)[\alpha-\beta, \ldots, \alpha-\beta]\right\| \leqq N^{k} M^{k+1}\left(\max \left\|a_{i}-b_{i}\right\|\right)^{k} .
$$

Thus,

Proposition 4.3. If $A$ is a free analytic algebra and $(X, \beta)$ is a representation of $A$ on a Banach space $X$, then for $\alpha$ in some neighborhood $V$ of $\beta$ the series

(4.8) $u(\beta)+\Delta u(\beta, \beta)[\alpha-\beta]+\cdots+\Delta^{k} u(\beta, \ldots, \beta)[\alpha-\beta, \ldots, \alpha-\beta]+\cdots$

converges absolutely and uniformly (on $V$ ) to $u(\alpha)$.

An alternate form for (4.8) is the following: Let $\alpha=\left(b_{1}+\lambda_{1} c_{1}, \ldots\right.$, $\left.b_{n}+\lambda_{n} c_{n}\right), \beta=\left(b_{1}, \ldots, b_{n}\right)$, and $\gamma=\left(c_{1}, \ldots, c_{n}\right)$, then the scalars $\lambda_{i}$ pull out of the terms of (4.8) to yield a power series in $\lambda_{1}, \ldots, \lambda_{n}$ with operator coefficients determined by the expressions $\Delta^{k} u(\beta, \ldots, \beta)[\gamma, \gamma, \ldots, \gamma]$.

The form of the power series (4.8) suggests that we attempt to construct 
free analytic algebras which are power series algebras with "coefficients" which lie in multiple tensor products of an operator algebra $L(X)$ or, more generally, some "coefficient" Banach algebra $B$. Specifically, for a Banach algebra $B$ and an $n$-tuple $r=\left(r_{1}, \ldots, r_{n}\right)$ of positive numbers, let $U_{r}(B)$ be the algebra consisting of all series of the form $\sum_{k=0}^{\infty} f_{k}$ with

$$
f_{k}=\sum_{|\sigma|=k} g_{\sigma} \in \sum_{\sigma=\left(j_{1}, \ldots, j_{k}\right)} B e_{j_{1}} B e_{j_{2}} \cdots B e_{j_{k}} B
$$

and $\sum_{k} \sum_{\sigma} t_{\sigma}\left\|g_{\sigma}\right\|<\infty$ for each tuple $t=\left(t_{1}, \ldots, t_{k}\right)$ with $t_{i}<r_{i}$, where $t_{\sigma}=t_{j_{1}} t_{j_{2}} \cdots t_{j_{k}}$. Elements of this algebra are multiplied in the obvious way.

We are attempting to construct a free analytic algebra with $a_{1}+e_{1}, \ldots, a_{n}+e_{n}$ as generators for some set $a_{1}, \ldots, a_{n} \in B$. There are maps $\Delta_{1}, \ldots, \Delta_{n}$ from $U_{r}(B)$ to $U_{r}(B) \hat{\otimes} U_{r}(B)$ which satisfy (a) and (b) of Definition 1.1. These are defined by

$$
\Delta_{l}\left(u_{0} e_{j_{1}} \cdots u_{k-1} e_{k} u_{k}\right)=\sum_{i} \delta_{l j_{i}}\left(u_{0} e_{j_{1}} \cdots e_{j_{i-1}}\right) \otimes\left(u_{j_{i}} e_{j_{i+1}} \cdots e_{j_{k}} u_{j_{k}}\right)
$$

in other words, by letting $\Delta_{l}$ be zero on terms of degree zero and then by summing over all ways of replacing an $e_{l}$ by $1 \otimes 1$ in higher degree terms.

The set of maps $\Delta_{1}, \ldots, \Delta_{n}$ defined by (4.9) certainly does not satisfy (c) of Definition 1.1. However, (c) is satisfied by $\Delta_{1}, \ldots, \Delta_{n}$ if we restrict to a certain subalgebra $P_{r}(B)$ of $U_{r}(B)$. The restrictions defining $P_{r}(B)$ are suggested by (4.5). They are

$$
\delta^{i} f_{k-1}=\theta^{i} f_{k} \text { for } i=1, \ldots, k \text { and } k=1,2, \ldots
$$

where

$$
\begin{aligned}
\delta^{i}\left(u_{0} e_{j_{1}}\right. & \left.\ldots e_{j_{k-1}} u_{k-1}\right) \\
& =u_{0} e_{j_{1}} \ldots u_{i-2} e_{j_{i-1}}\left(u_{i-1} \otimes 1-1 \otimes u_{i-1}\right) e_{j_{i}} u_{j_{i}} \ldots e_{j_{k-1}} u_{j_{k-1}}
\end{aligned}
$$

and

$$
\theta^{i}\left(u_{0} e_{j_{1}} \ldots e_{j_{k}} u_{k}\right)=u_{0} e_{j_{1}} \ldots u_{j_{i-1}}\left(a_{j_{1}} \otimes 1-1 \otimes a_{j_{i}}\right) u_{j_{i}} \ldots e_{j_{k}} u_{j_{k}} .
$$

It turns out that the maps $\Delta_{1}, \ldots, \Delta_{n}$ do satisfy (c) of Definition 1.1 when restricted to $P_{r}(B)$. Now, each $\Delta_{i}$ maps $P_{r}(B)$ into $U_{r}(B) \hat{\otimes} U_{r}(B)$, but does it define a map of $P_{r}(B)$ into $P_{r}(B) \hat{\otimes} P_{r}(B)$ ? It is possible to prove that the conditions defining $P_{r}(B)$ are satisfied in each factor by an element of $U_{r}(B) \hat{\otimes} U_{r}(B)$ in the image of $\Delta_{i}$, but this is not enough to prove that such an element actually lies in $P_{r}(B) \hat{\otimes} P_{r}(B)$. The difficulty is this: Given closed subspaces $Y_{i}$ of topological vector spaces $X_{i}(i=1,2)$, the projective tensor product topology for $Y_{1} \otimes Y_{2}$ may be strictly stronger than the projective tensor product topology for $X_{1} \otimes X_{2}$ restricted to $Y_{1} \otimes Y_{2}$. Hence, the completed space $Y_{1} \hat{\otimes} Y_{2}$ may fail to have a closed image in 
$X_{1} \hat{\otimes} X_{2}$ (cf. [12, Remark 43.2]). There is no difficulty if $Y_{1}$ and $Y_{2}$ are nuclear spaces (cf. [13,50.1]) or if they are direct summands of $X_{1}$ and $X_{2}$. Unfortunately, neither of these conditions seems likely to hold in our situation.

We have thus far been unable to see a way out of the above difficulty in general. However, in the case where $B$ is a finite-dimensional matrix algebra and $a_{1}, \ldots, a_{n} \in B$ have the property that only multiples of the identity commute with each $a_{i}$, we believe that for small enough $r, P_{r}(B)$ is a direct summand of $U_{r}(B)$ and, hence, is a localization (in fact, a free analytic algebra), and also has the property that every $b \in B$ is the image of some element of $P_{r}(B)$ under the representation generated by the tuple $\alpha=\left(a_{1}, \ldots, a_{n}\right)$ (i.e., the representation $\left.\sum_{k=0}^{\infty} f_{k} \rightarrow f_{0}\right)$. The reason we believe this is that, in this situation, we can define, for each $i$, a map which assigns to a tuple $f_{0}, f_{1}, \ldots, f_{l}$ satisfying (4.10) for $1 \leqq k \leqq l$, an element $f_{l+1}$ so that (4.10) is satisfied for $k=l+1$ as well. If this map satisfies appropriate bounds it can be used to construct $\sum f_{k} \in P_{r}(B)$ with a given first term $f_{0}$ and also to define a map from $U_{r}(B)$ to $P_{r}(B)$ which is the identity on $P_{r}(B)$. This would show that $P_{r}(B)$ was a direct summand of $U_{r}(B)$ and eliminate, for this case, our difficulties with the proof that $P_{r}(B)$ is a localization. We believe that our map does satisfy the right bounds for small enough $r$ but have not checked all the details.

For infinite-dimensional $B$, the problem of salvaging something out of the above construction seems quite difficult.

5. The implicit function theorem. If $u$ is a $C^{\infty}$ function on a domain in $R^{n}$ (or an analytic function on a domain in $C^{n}$ ) and if the partial derivative of $u$ with respect to one coordinate never vanishes on the zero set of $u$, then the ordinary implicit function theorem states that the zero set of $u$ is an $(n-1)$-dimensional manifold with a global coordinate system given by the remaining coordinate functions. For an $m$-tuple of functions, a similar statement holds with the Jacobian matrix relative to $m$ of the coordinates playing the role of the partial derivative. For localizations of $F_{n}$, there is a complete analogue of this form of the implicit function theorem. Here the two-sided ideal generated by an element $u$ plays the role of the "zero set."

Thus, let $A$ be a localization of $F_{n}$ with generators $z_{1}, \ldots, z_{n}$. Let $w$ be an element of $A, I$ the two-sided ideal generated by $w$, and $\tilde{I}$ the kernel of the map $A \hat{\otimes} A \rightarrow A / I \hat{\otimes} A / I$ (note that $\tilde{I}$ contains $I \hat{\otimes} A+A \hat{\otimes} I$ ). As before, we consider $A \hat{\otimes} A$ to be an algebra with operation

$$
(u \otimes v) *\left(u^{\prime} \otimes v^{\prime}\right)=u u^{\prime} \otimes v^{\prime} v .
$$

Note that $\tilde{I}$ is a two-sided ideal of $A \hat{\otimes} A$.

By Definition 1.1 we have $\delta w=\left(\Delta_{1} w\right) * \delta z_{1}+\cdots+\left(\Delta_{n} w\right) * \delta z_{n}$. Now 
suppose $\Delta_{n} w$ has an inverse modulo $\tilde{I}$; that is, suppose there is an element $g \in A \hat{\otimes} A$ such that $g *\left(\Delta_{n} w\right)-1 \otimes 1 \in \tilde{I}$ and $\left(\Delta_{n} w\right) * g-1 \otimes 1 \in \tilde{I}$. We then set

$$
\bar{\Delta}_{i} u=\Delta_{i} u-\left(\Delta_{n} u\right) * g * \Delta_{i} w \quad \text { for } i=1, \ldots, n-1 .
$$

It is clear that the $\bar{\Delta}_{i}$ 's satisfy (a) and (b) of Definition 1.1 for the variables $z_{1}, \ldots, z_{n-1}$. Furthermore,

$$
\begin{aligned}
\bar{\Delta}_{1} u * \delta z_{1}+ & \cdots+\bar{\Delta}_{n-1} u * \delta z_{n-1} \\
= & \Delta_{1} u * \delta z_{1}+\cdots+\Delta_{n-1} u * \delta z_{n-1} \\
& -\Delta_{n} u * g *\left[\Delta_{1} w * \delta z_{1}+\cdots+\Delta_{n-1} w * \delta z_{n-1}\right] \\
= & \Delta_{1} u * \delta z_{1}+\cdots+\Delta_{n-1} u * \delta z_{n-1}-\Delta_{n} u * g *\left[\delta w-\Delta_{n} w * \delta z_{n}\right] \\
= & \Delta_{1} u * \delta z_{1}+\cdots+\Delta_{n-1} u * \delta z_{n-1}+\Delta_{n} u *\left(g * \Delta_{n} w\right) * \delta z_{n} \\
& -\Delta_{n} u * g * \delta w
\end{aligned}
$$

which is equivalent $\bmod \tilde{I}$ to $\delta u$. Thus, $\bar{\Delta}_{1}, \ldots, \bar{\Delta}_{n-1}$ satisfy (c) of Definition 1.1 for $z_{1}, \ldots, z_{n-1}$ modulo the ideal $\tilde{I}$.

Note that $\bar{\Delta}_{i} w=\Delta_{i} w-\Delta_{n} w * g * \Delta_{i} w \in \tilde{I}$ and that (b) of Definition 1.1 implies that, in fact, $\bar{\Delta}_{i}$ maps all of $I$ into $\tilde{I}$. It follows that $\bar{\Delta}_{i}$ defines a map $\tilde{\Delta}_{i}: A / I \rightarrow A / I \hat{\otimes} A / I$ for $i=1, \ldots, n-1$, such that (a), (b), and (c) of Definition 1.1 are satisfied relative to the variables $z_{1}, \ldots, z_{n-1}$. In other words, $A / I$ is a localization of $F_{n-1}$ with generators $z_{1}, \ldots, z_{n-1}$.

It is not difficult to see that if the $\Delta_{i}$ 's satisfy the continuity condition of Definition 3.1 relative to some sequence of seminorms defining the topology of $A$, then the $\tilde{\Delta}_{i}$ 's satisfy the condition relative to the corresponding induced family of seminorms on $A / I$. Hence, if $A$ is a free analytic algebra then so is $A / I$.

Now suppose $w_{1}, \ldots, w_{m}$ are elements of $A$ generating a two-sided ideal $I$, and that the $(n-k) \times m$ matrix $J=\left(\Delta_{k+j} w_{i}\right)_{i j}(i=1, \ldots, m, j=1, \ldots$, $n-k)$ is invertible modulo the ideal $\tilde{I} \subset A \hat{\otimes} A$ corresponding to $I$; that is, $J$ defines an invertible operator from $\bigoplus^{n-k}(A / I \hat{\otimes} A / I)$ to $\oplus^{m}(A / I \hat{\otimes} A / I)$. It follows from the fact that this operator is a right $A \hat{\otimes} A$-module homomorphism that its inverse must be determined by an $m \times(n-k)$-matrix with entries from $A \hat{\otimes} A$. This matrix then plays the role of $g$ in a vector-matrix version of our previous argument. Such an argument establishes

Proposition 5.1. Let $A$ be a localization (free analytic algebra) with generators $z_{1}, \ldots, z_{n}$. If $w_{1}, \ldots, w_{m} \in A, I$ is the two-sided ideal they generate, and $\tilde{I}$ is the kernel of $A \hat{\otimes} A \rightarrow A / I \hat{\otimes} A / I$, then $A / I$ is a localization (free analytic algebra) with generators $z_{1}, \ldots, z_{k}$ provided the matrix 
$J=\left(\Delta_{k+j} w_{i}\right)_{i j}$ is invertible modulo $\tilde{I}$.

Note that since we are working with noncommutative algebras a matrix $J$ like the one above can be invertible without being square. We shall call the matrix $J=\left(\Delta_{k+j} w_{i}\right)_{i j}$ the Jacobian matrix for $w_{1}, \ldots, w_{m}$ and $z_{k+1}, \ldots, z_{n}$.

We conclude this section with three examples. The first two show how Proposition 5.1 can be used to construct free analytic algebras in which certain equations have solutions.

EXAMPLE 1. For some multi-radius $r=\left(r_{1}, \ldots, r_{n}\right)$, let $\mathscr{F}_{n}(r)$ be the power series algebra of $\S 3$. We wish to embed $\mathscr{F}_{n}(r)$ in a new free analytic algebra (on the same generators) in which a given element $v \in \mathscr{F}_{n}(r)$ has an inverse. This is possible provided for some $r_{n+1}, r_{n+2}$ the ideal $I$ generated by $w_{1}=v z_{n+1}-1$ and $w_{2}=z_{n+2} v-1$ in $\mathscr{F}_{n+2}\left(r^{\prime}\right)\left(r^{\prime}=\left(r_{1}, \ldots, r_{n+2}\right)\right)$ is proper. In fact, in this case the Jacobian matrix for $\left(w_{1}, w_{2}\right)$ and $\left(z_{n+1}, z_{n+2}\right)$ is

$$
\left(\begin{array}{cc}
v \otimes 1 & 0 \\
0 & 1 \otimes v
\end{array}\right)
$$

which has left inverse

$$
\left(\begin{array}{cc}
z_{n+2} \otimes 1 & 0 \\
0 & 1 \otimes z_{n+1}
\end{array}\right)
$$

modulo $\tilde{I}$ and right inverse

$$
\left(\begin{array}{cc}
1 \otimes z_{n+1} & 0 \\
0 & z_{n+2} \otimes 1
\end{array}\right)
$$

modulo $\tilde{I}$. It follows that either one of these is actually an inverse modulo $\tilde{I}$ and, hence, that $\mathscr{F}_{n+2}\left(r^{\prime}\right) / I$ is a free analytic algebra with generators $z_{1}, \ldots, z_{n}$. Clearly, the elements $z_{n+1}$ and $z_{n+2}$ are equal modulo $I$ and provide an inverse for $v$.

EXAMPLE 2. Again we begin with $\mathscr{F}_{n}(r)$. However, this time we wish to construct an embedding of $\mathscr{F}_{n}(r)$ in a free analytic algebra such that for a given $u, v \in \mathscr{F}_{n}(r)$ the system of equations

$$
u x+v y=1, \quad x u=y v=1, \quad x v=y u=0
$$

has a solution $x, y$. This is possible provided for some $r_{n+1}, r_{n+2}, r_{n+3}$, $r_{n+4}$ the ideal $I$ generated by

$$
\begin{aligned}
& w_{1}=u z_{n+1}+v z_{n+2}-1, \quad w_{2}=z_{n+3} u-1, \\
& w_{3}=z_{n+3} v, \quad w_{4}=z_{n+4} v-1, \quad w_{5}=z_{n+4} u
\end{aligned}
$$

is proper in $\mathscr{F}_{n+4}\left(r^{\prime}\right)\left(r^{\prime}=\left(r_{1}, \ldots, r_{n+4}\right)\right)$. In fact, here the Jacobian for 
$\left(w_{1}, \ldots, w_{5}\right)$ and $\left(z_{n+1}, \ldots, z_{n+4}\right)$ is

$$
\left(\begin{array}{cccc}
u \otimes 1 & v \otimes 1 & 0 & 0 \\
0 & 0 & 1 \otimes u & 0 \\
0 & 0 & 1 \otimes v & 0 \\
0 & 0 & 0 & 1 \otimes v \\
0 & 0 & 0 & 1 \otimes u
\end{array}\right)
$$

which has left and right inverses modulo $\tilde{I}$ given by

$$
\left(\begin{array}{ccccc}
z_{n+3} \otimes 1 & 0 & 0 & 0 & 0 \\
z_{n+4} \otimes 1 & 0 & 0 & 0 & 0 \\
0 & 1 \otimes z_{n+1} & 1 \otimes z_{n+2} & 0 & 0 \\
0 & 0 & 0 & 1 \otimes z_{n+2} & 1 \otimes z_{n+1}
\end{array}\right)
$$

and

$$
\left(\begin{array}{ccccc}
z_{n+1} \otimes 1 & 0 & 0 & 0 & 0 \\
z_{n+2} \otimes 1 & 0 & 0 & 0 & 0 \\
0 & 1 \otimes z_{n+3} & 1 \otimes z_{n+4} & 0 & 0 \\
0 & 0 & 0 & 1 \otimes z_{n+4} & 1 \otimes z_{n+3}
\end{array}\right)
$$

It follows that modulo $\tilde{I}$ these matrices agree and determine an inverse for the Jacobian. Thus, by Proposition 5.1, $\mathscr{F}_{n+4}\left(r^{\prime}\right) / I$ is a free analytic algebra in which the elements $x=z_{n+1}=z_{n+3}$ and $y=z_{n+2}=z_{n+4}$ provide a solution to (5.1).

Obviously, the above procedure can be extended to obtain free analytic algebras and elements $u_{1}, \ldots, u_{k}$ for which the system

$$
u_{1} x_{1}+\cdots+u_{n} x_{n}=1, \quad x_{i} u_{j}=\delta_{i j}
$$

has a solution. Note that such an algebra can have no finite-dimensional representations since the system of equations (5.2) can never be satisfied among operators on a finite-dimensional space $H$ (since (5.2) means exactly that the map $\oplus^{k} H \rightarrow H$, determined by $h_{1} \otimes \cdots \otimes h_{k} \rightarrow$ $u_{1} h_{1}+\cdots+u_{k} h_{k}$, is invertible).

One choice for the tuple $\left(u_{1}, \ldots, u_{k}\right)$ in (5.2) is the tuple of generators $\left(z_{1}, \ldots, z_{n}\right)$ of $\mathscr{F}_{n}(r)$. In this case it is in fact possible to embed $\mathscr{F}_{n}(r)$ in a free analytic algebra with generators $z_{1}, \ldots, z_{n}$ such that (5.2) has a solution. An explicit construction of such an algebra was carried out in [11]. The construction amounts essentially to what was done above in the two variable case. Here the problem of proving that the ideal $I$ is proper for an appropriate choice of $\left(r_{n+1}, r_{n+2}, \ldots\right)$ is handled by demonstrating an explicit Banach space representation of $\mathscr{F}_{n}(r)$ )in which the equation 
(5.2) has a solution for operators $x_{1}, \ldots, x_{n}$ on the space. The norms of these operators yield choices for $\left(r_{n+1}, \ldots\right)$ such that the representation of $\mathscr{F}_{n}(r)$ extends to a nontrivial representation of the new algebra, thus proving that the new algebra is nontrivial and $I$ is proper.

The reason for being interested in equations like (5.2) is that the solvability of $(5.2)$ for $\left(u_{1}, \ldots, u_{k}\right)$ seems to provide the "correct" definition (in the context of several free variables) of the statement that the tuple $\left(u_{1}, \ldots, u_{k}\right)$ is nonsingular. We shall discuss this point in more detail in $\$ 7$.

EXAMPLE 3. Let $u_{1}, \ldots, u_{k}$ be elements of $F_{n}$ and $r_{1}, \ldots, r_{k}>0$. In the algebra $\mathscr{F}_{n+k}(r) \quad\left(r=\left(\infty, \ldots, \infty, r_{1}, \ldots, r_{k}\right)\right)$, we consider the ideal $I$ generated by $w_{1}=z_{n+1}-u_{1}, \ldots, w_{k}=z_{n+k}-u_{k}$. Note that the Jacobian is the $k \times k$ identity matrix

$$
\left(\begin{array}{cccc}
1 \otimes 1 & 0 & \cdots & 0 \\
0 & 1 \otimes 1 & \cdots & 0 \\
\vdots & \vdots & & \vdots \\
0 & 0 & \cdots & \otimes
\end{array}\right)
$$

which is invertible. Hence, if $I$ is proper then $\mathscr{F}_{n+k}(r) / I$ is a free analytic algebra on the generators $z_{1}, \ldots, z_{n}$. Note that in each of the seminorms defining the topology of this algebra, the elements $u_{1}, \ldots, u_{k}$ have norms less than or equal to the corresponding numbers $r_{1}, \ldots, r_{k}$. Algebras of this type are the appropriate free analogues of algebras of analytic functions on polynomial polyhedra.

6. A compactness result. The free analytic algebras on a single generator (the algebras $\mathcal{O}(U)$ ) are all nuclear spaces. However, it appears that free analytic algebras on more than one generator are never nuclear. Certainly, the algebras $\mathscr{F}_{n}(r)$ for $n>1$ are not nuclear (cf. $\left.[11, \S 6]\right)$. They are, however, Montel spaces (bounded sets are precompact). We suspect that all free analytic algebras are Montel spaces. In fact, for the distinguished family of seminorms $\left\{\rho_{i}\right\}$ of Definition 3.1, we conjecture that the bonding maps $A_{i} \rightarrow A_{i-1}$, of the corresponding inverse limit system must be compact operators. This would imply that $A=\lim _{A_{i}}$ was Montel.

If $A=\varliminf_{i} A_{i}$ is a free analytic algebra, with the $A_{i}$ 's the Banach algebras of $\S 3$, then recall that $A_{i} \gg A_{i-1}$ for each $i$. Now if $A \rightarrow B$ is a homomorphism, with dense range, of $A$ into a Banach algebra $B$ and $u \rightarrow u(\alpha)$ determines a representation of $F_{n}$ on a Banach space $X$, then the relation $B \gg(X, \alpha)$ implies that $u \rightarrow u(\alpha)$ extends all the way to a representation $\varphi: B \rightarrow L(X)$ of $B$ on $X$ (Proposition 2.1). We suspect that the relation $B \gg(X, \alpha)$ is strong enough to force $\varphi$ to be compact. If this were true it would certainly imply that the maps $A_{i} \rightarrow A_{i-1}$ in the inverse limit 
system for $A$ were compact.

Now given $B$ and $(X, \alpha)$ as above, the relation $B \gg(X, \alpha)$ implies the exactness of the sequence (2.3) with $B=M$ and the fact that $\theta: \oplus^{n} B \hat{\otimes} X \rightarrow B \hat{\otimes} X$ has a left inverse. It follows that there is a constant $K>0$ such that, for each $u \in B, x \in X$ with $\|u\| \leqq 1,\|x\| \leqq 1$, there are $f_{1}, \ldots, f_{n} \in B \hat{\otimes} X$ with $\left\|f_{i}\right\| \leqq K$ and

$$
u \otimes x-1 \otimes u x=f_{1} * \delta z_{1}+\cdots+f_{n} * \delta z_{n} .
$$

If $g_{i}=(\varphi \otimes 1), f_{i} \in L(X) \hat{\otimes} X$ and $c \rightarrow g_{i}[c]$ is the map from $L(X)$ to $X$ determined by inserting $c$ between factors in the tensor product, then (since $\varphi\left(z_{i}\right)=a_{i}$ )

$$
\varphi(u) c x-c \varphi(u) x=g_{1}\left[a_{i} c-c a_{i}\right]+\cdots+g_{n}\left[a_{n} c-c a_{n}\right],
$$

which implies that, for $K^{\prime}=\|\varphi\| K$,

(6.1) $\|b c-c b\| \leqq K^{\prime}\left[\left\|a_{1} c-c a_{1}\right\|+\cdots+\left\|a_{n} c-c a_{n}\right\|\right] \quad$ for $c \in L(X)$,

for all $b$ in the image under $\varphi$ of the unit ball of $B$.

The condition (6.1) on a family of operators $b$ has something in common with a uniform Lipschitz condition for a family of ordinary functions. We feel that something like the Ascoli-Arzela Theorem is at work here and that a bounded family in $L(X)$, satisfying (6.1) uniformly, must be precompact in the norm topology. We cannot prove this, but we can prove that such a family is strongly precompact if $X$ is a Hilbert space. Our proof depends on the following:

Proposition 6.1. Let $X$ be a Hilbert space and $a_{1}, \ldots, a_{n}$ any finite set of operators in $L(X)$. Then, for any $\varepsilon>0$ and any finite-dimensional subspace $M \subset X$, there is a finite rank operator $c \in L(X)$ with $c=1$ on $M$ and $\left\|a_{i} c-c a_{i}\right\|<\varepsilon$ for $i=1, \ldots, n$.

Proof. Let $S$ be the set of all finite rank operators $a$ on $X$ for which $\|a\| \leqq 1$ and $a=1$ on $M$. If $L_{c}(X)$ denotes the space of compact operators on $X$, we define a map $\varphi: L_{c}(X) \rightarrow \bigoplus^{n} L_{c}(X)$ by

$$
\varphi(c)=\bigoplus_{i=1}^{n}\left(a_{i} c-c a_{i}\right)
$$

Now the set $S$ is convex and so its image $\varphi(S)$ under $\varphi$ is a convex subset of the Banach space $L_{c}(X)$. The conclusion of the proposition is precisely that the norm closure of $\varphi(s)$ contains zero.

Suppose that zero is not in the closure of $\varphi(s)$. Then there exists a bounded linear functional $F$ on $\bigoplus^{n} L_{c}(X)$ such that $\operatorname{Re} F(\varphi(S)) \geqq k>0$. Now comes the crucial fact about Hilbert space: The adjoint space of $L_{c}(X)$ is the space $L_{T}(X)$ of trace class operators with trace norm, where the pairing is defined by $(b, c) \rightarrow \operatorname{tr}(b c)$ (cf. [12, §49]). It follows that the 
functional $F$, above, has the form $F\left(c_{1}, \ldots, c_{n}\right)=\operatorname{tr}\left(b_{1} c_{1}\right)+\cdots+\operatorname{tr}\left(b_{n} c_{n}\right)$ for some set $b_{1}, \ldots, b_{n}$ of trace class operators. Thus, for this set of operators we have

$$
\operatorname{Re}\left(\operatorname{tr}\left[b_{1}\left(a_{1} c-c a_{1}\right)\right]+\cdots+\operatorname{tr}\left[b_{n}\left(a_{n} c-c a_{n}\right)\right]\right) \geqq k>0,
$$

for all $c \in S$. Furthermore, since the finite rank operators are dense in the space of trace class operators with trace norm, we can assume without loss of generality that the $b_{i}$ 's are actually of finite rank.

Now if $N$ is the linear span of the ranges of the operators $b_{i} a_{i}-a_{i} b_{i}$ and the space $M$, then the orthogonal projection $p$ on $N$ is an element of $S$. We choose $c=p$ in (6.2) and note that, for each $i$,

$$
\operatorname{tr}\left(b_{i}\left(a_{i} p-p a_{i}\right)\right)=\operatorname{tr}\left(p\left(b_{i} a_{i}-a_{i} b_{i}\right)\right)=\operatorname{tr}\left(b_{i} a_{i}-a_{i} b_{i}\right)=0 .
$$

The resulting contradiction shows that zero is in the closure of $\varphi(S)$.

Proposition 6.2. If $X$ is a Hilbert space, $K>0$, and $a_{1}, \ldots, a_{n} \in L(X)$, then the set $T$ of all operators $b \in L(X)$ with $\|b\| \leqq K$ and

$$
\|b c-c b\| \leqq K \sum_{i}\left\|a_{i} c-c a_{i}\right\| \quad \text { for all } c \in L(X)
$$

is precompact in the strong operator topology of $L(X)$.

Proof. Since $T$ is bounded, it is precompact in the weak operator topology. We shall show that the weak and strong operator topologies coincide on $T$ due to (6.3).

For a given $x \in X$, we choose a sequence $\left\{c_{j}\right\}$ of finite rank operators with $c_{j} x=x,\left\|c_{j}\right\|=1$ and $\left\|a_{i} c_{j}-c_{j} a_{i}\right\|<1 / j$ for each $i, j$ (using Proposition 6.1). Then, by (6.3), we have

$$
\left\|b c_{j}-c_{j} b\right\|<n K / j \quad \text { for } i=1,2,3, \ldots, \text { and } b \in T .
$$

It follows that for $b, b^{\prime} \in T$ we have

$$
\left\|b x-b^{\prime} x\right\|=\left\|\left(b c_{j}-b^{\prime} c_{j}\right) x\right\| \leqq 2 n K / j+\left\|c_{j}\left(b x-b^{\prime} x\right)\right\| .
$$

However, since each $c_{j}$ has finite rank, the map $b \rightarrow\left\|c_{j} b x\right\|$ is continuous in the weak operator topology for each $j$. It follows from this and (6.4) that if $b_{\alpha} \rightarrow b$ in $T$ in the weak operator topology then $b_{\alpha} x \rightarrow b x$ in norm. Hence, weak operator convergence implies strong operator convergence in $T$. This completes the proof.

If the above result could be proved for general Banach spaces $X$, then it would imply the compactness of the maps $A_{i} \rightarrow A_{i-1}$ for a free analytic algebra $A$. In fact, with $X=A_{i-1}$ the relation $A \gg A_{i-1}$ implies that the image of the unit ball of $A_{i}$ consists of a set $E$ of elements of $A_{i-1}$ satisfying (6.3) when considered as a set of operators on $A_{i-1}$ via left multiplication. Thus, if Proposition 6.2 held for $X=A_{i-1}$, the set $E$, considered as a set 
of operators, would have to be precompact in the strong operator topology. However, since $A_{i-1}$ has an identity, the strong operator topology coincides with the norm topology for left multiplication operators.

The relation expressed by (6.3) between an operator $b$ and a tuple of operators $a_{1}, \ldots, a_{n}$ strikes us as interesting in itself, independently of its relation to the study of free analytic algebras. It appears to be considerably stronger than simply saying that $c$ belongs to the second commutant of the set $\left\{a_{1}, \ldots, a_{n}\right\}$. It is tempting to let this relation define the statement " $b$ is a Lipschitz function of the operators $a_{1}, \ldots, a_{n}$ "; however, for the case of a single selfadjoint operator $a$ of the form $a f(\lambda)=\lambda f(\lambda)$ on $L^{2}(\mu)$ for a compactly supported measure $\mu$ on the line, the relation seems to imply somewhat more than the fact that $b$ has the form $b f(\lambda)=g(\lambda) f(\lambda)$ for some Lipschitz function $g$ on the support of $\mu$.

7. Resolvents and the functional calculus. For a localization $F_{n} \rightarrow A$ and a left $F_{n}$-module $X$, the functional calculus problem is the problem of deciding whether or not the corresponding representation of $F_{n}$ on $X$ extends to a representation of $A$. According to Proposition 2.2, an extension exists if and only if $A \gg X$, when $A$ is considered an augmented right $F_{n}$-module. Here we shall present some results which make plausible the following formula for attacking the functional calculus problem: Begin with an augmented right $F_{n}$-module satisfying $M \gg X$, and then modify $M$ by a sequence of steps which preserve this relation and end with $A$. These "allowable" modifications of augmented right $F_{n}$-modules involve a relation " $\perp$ " between right and left $F_{n}$-modules which is complementary to " $\gg$ " and is related to the resolvent set of single variable spectral theory.

Recall from $\S 2$ that if $M$ is a right $F_{n}$-module and $X$ a left $F_{n}$-module, then there is a map $\theta: \bigoplus^{n}(M \otimes X) \rightarrow M \hat{\otimes} X$ defined in terms of the generators $z_{1}, \ldots, z_{n}$ of $A$ by

$$
\theta\left(f_{1} \oplus \cdots \oplus f_{n}\right)=f_{1} * \delta z_{1}+\cdots+f_{n} * \delta z_{n},
$$

where $(m \otimes x) *(u \otimes v)=m u \otimes v x$ and $\delta u=u \otimes 1-1 \otimes u$. If $M$ is augmented with distinguished vector $e$, then the condition that $M \gg X$ is equivalent to the statement that the maps $\theta$ and $x \rightarrow e \otimes x: X \rightarrow M \otimes X$ have left inverses and the direct sum of their images is $M \hat{\otimes} X$. Another way of saying this is that there is a map $\varphi: M \otimes X \rightarrow X$ for which the sequence

$$
0 \rightarrow \stackrel{n}{\oplus}(M \hat{\otimes} X) \stackrel{\theta}{\rightarrow} M \hat{\otimes} X \stackrel{\varphi}{\rightarrow} X \rightarrow 0
$$

is exact and split.

Definition 7.1. If $M$ is a right $F_{n}$-module and $X$ a left $F_{n}$-module, then we shall write $M \perp X$ if the map $\theta: \bigoplus^{n}(M \hat{\otimes} X) \rightarrow M \hat{\otimes} X$ is invertible. The class of modules $M$ satisfying $M \perp X$ will be called the resolvent 
class for $X$.

The importance of the notion " $\perp$ " lies in the fact that the following statements are almost true: If $L$ is a submodule of an augmented module $M$ and $L \perp X$, then $M \gg X$ if and only if $M / L \gg X$; similarly, if $L$ is an augmented submodule of the augmented module $M$ and $N=M / L$ satisfies $N \perp X$, then $M \gg X$ if and only if $L \gg X$.

The reason the above statements are only "almost" true is that the functor $(\cdot) \hat{\otimes} X$ does not, in general, preserve the exactness of short exact sequences. This is a very annoying difficulty that is basic to the subject. We give a brief description of the problem and then move on to the more complicated but correct versions of the above relations between " $\perp$ " and " $\gg "$.

Suppose $0 \rightarrow L \rightarrow^{\alpha} M \rightarrow^{\beta} N \rightarrow 0$ is a short exact sequence of topological vector spaces and continuous linear maps. If $X$ is a topological vector space then for the sequence

$$
0 \rightarrow L \hat{\otimes} X \stackrel{\alpha \otimes 1}{\longrightarrow} M \hat{\otimes} X \stackrel{\beta \otimes 1}{\longrightarrow} N \hat{\otimes} X \longrightarrow 0,
$$

$\beta \otimes 1$ has dense image and $\alpha \otimes 1$ has its image dense in $\operatorname{ker}(\beta \otimes 1)$ (cf. $[12, \S 43]$ ). If $M, N$ and $X$ are Fréchet spaces then $\beta \otimes 1$ is actually onto (cf. $[12, \S 43])$. However, even if all the spaces are Fréchet spaces (or even Banach spaces) $\alpha \otimes 1$ may fail to have closed image and it is not known whether or not it must be one to one (cf. [2, I, $\left.\left.\S 1, n^{\circ} 2\right]\right)$. There are several special conditions which can be imposed, each of which forces $\alpha \otimes 1$ to be a topological isomorphism onto its image and, hence, to have closed image equal to $\operatorname{ker}(\beta \otimes 1)$. One such condition is that $L$ or $X$ be nuclear (cf. [2, II, $\left.\S 3, \mathrm{n}^{\circ} 1\right]$ ); another is that $X$ be isomorphic to a space of the form $L^{1}(\mu)$ for some measure $\mu$, or that it have a topology defined by a family of seminorms for which the corresponding Banach spaces have this form (cf. [2, I, §2, $\left.\mathrm{n}^{\circ} 2\right]$ ); another is that $L$ be a topological direct summand of $M$ (i.e. that the sequence $0 \rightarrow L \rightarrow M \rightarrow N \rightarrow 0$ be split).

Proposition 7.1. Let $0 \rightarrow L \rightarrow^{\alpha} M \rightarrow^{\beta} N \rightarrow 0$ be a short exact sequence of right $F_{n}$-modules and let $X$ be a left $F_{n}$-module. Suppose that $L, M, N, X$ are Fréchet spaces and $\alpha \otimes 1: L \hat{\otimes} X \rightarrow M \hat{\otimes} X$ is a topological isomorphism onto its range (equivalently, $\alpha \otimes 1$ is one to one and has closed range). Then

(a) if $M$ and $N$ are augmented and $\beta$ preserves the distinguished vector, any two of $L \perp X, M \gg X$, and $N \gg X$ imply the third;

(b) if $L$ and $M$ are augmented and $\alpha$ preserves the augmentation, any two of $L \gg X, M \gg X$, and $N \perp X$ imply the third;

(c) any two of the relations $L \perp X, M \perp X$, and $N \perp X$ imply the third.

Proof. The hypotheses are sufficient to force 


$$
0 \rightarrow L \hat{\otimes} X \stackrel{\alpha \otimes 1}{\longrightarrow} M \hat{\otimes} X \stackrel{\beta \otimes 1}{\longrightarrow} N \hat{\otimes} X \rightarrow 0
$$

to be an exact sequence of Fréchet spaces. The proof then follows from an analysis of the commutative diagram

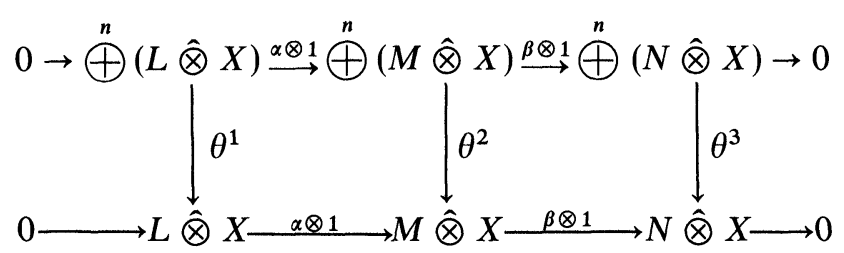

where $\theta^{1}, \theta^{2}$, and $\theta^{3}$ are the maps $\theta$ of (7.1) for $L \hat{\otimes} X, M \hat{\otimes} X$, and $N \hat{\otimes} X$, respectively. In fact, the invertibility of $\theta^{1}$ (or $\theta^{3}$ ) is equivalent to the fact that the other two maps have isomorphic kernels and isomorphic cokernels (via the maps induced by $\beta \otimes 1$ (or $\alpha \otimes 1)$ ). This, together with the open mapping theorem for Fréchet spaces, yields all three parts of the proposition.

The relations $M \perp X$ and $M \gg X$ also have certain invariance properties relative to the second variable $X$. The first of these is proved by simple diagram chasing in the following:

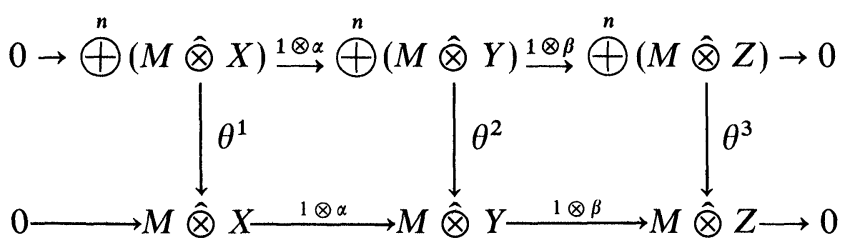

This is the result.

Proposition 7.2. Let $0 \rightarrow X \rightarrow^{\alpha} Y \rightarrow^{\beta} Z \rightarrow 0$ be a short exact sequence of left $F_{n}$-modules and $M$ a right $F_{n}$-module. Suppose $X, Y, Z$, and $M$ are Fréchet spaces and the map $1 \otimes \alpha: M \hat{\otimes} X \rightarrow M \hat{\otimes} Y$ is one to one and has closed range. Then

(a) any two of $M \perp X, M \perp Y$, and $M \perp Z$ imply the third;

(b) if $M$ is augmented, then any two of $M \gg X, M \gg Y, M \gg Z$ imply the third.

Now suppose that $\alpha: X \rightarrow Y$ is a left $F_{n}$-module homomorphism. Then

$$
K=\operatorname{Ker}(\alpha) \text { and } L=\overline{\operatorname{Im}(\alpha)}
$$

are closed submodules of $X$ and $Y$ respectively. Furthermore, if the induced map $M \hat{\otimes} K \rightarrow M \hat{\otimes} X$ is a topological isomorphism, then $M \hat{\otimes} K$ may be identified with the kernel of $1 \otimes \alpha: M \hat{\otimes} X \rightarrow M \hat{\otimes} Y$. Similarly, if $M \hat{\otimes} L \rightarrow M \hat{\otimes} Y$ is a topological isomorphism, then $M \hat{\otimes} L$ 
may be identified with the closure of the image of $1 \otimes \alpha: M \hat{\otimes} X \rightarrow M \hat{\otimes} Y$. The diagram

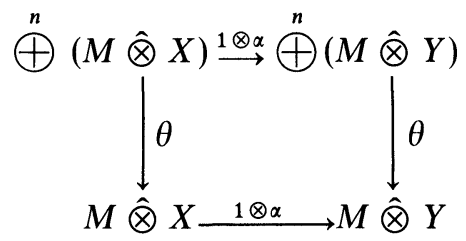

is commutative, and so if $\theta$ is invertible for both $M \hat{\otimes} X$ and $M \hat{\otimes} Y$, its inverse commutes with $1 \otimes \alpha$. This implies that $\theta^{-1}$ maps the kernel and closure of the image of $1 \otimes \alpha: M \hat{\otimes} X \rightarrow M \hat{\otimes} Y$ into the kernel and closure of the image of $1 \otimes \alpha: \bigoplus^{n}(M \otimes X) \rightarrow \bigoplus^{n}(M \otimes Y)$, respectively, and, hence, $\theta$ is invertible for these spaces as well.

A similar analysis works for the map $\mathscr{N}$ of $\S 2$ as well. We conclude that

Proposition 7.3. If $\alpha: X \rightarrow Y$ is a left $F_{n}$-module homomorphism, and $M$ is a right $F_{n}$-module for which $M \perp X, M \perp Y$ (or $M$ is augmented and $M \gg X, M \gg Y)$, then

(a) if $M \hat{\otimes}(\operatorname{Ker}(\alpha)) \rightarrow M \hat{\otimes} X$ is a topological isomorphism, we have $M \perp \operatorname{Ker}(\alpha)($ or $M \gg \operatorname{Ker}(\alpha))$;

(b) if $M \hat{\otimes}(\overline{\operatorname{Im}(\alpha)}) \rightarrow M \hat{\otimes} Y$ is a topological isomorphism we have $M \perp \overline{(\operatorname{Im}(\alpha))}($ or $M \gg \operatorname{Im}(\alpha))$.

The relations “ $\perp$ " and " $\gg$ " were introduced and discussed in [11] for more general base algebras (other than just $F_{n}$ ). Propositions 7.1 and 7.2 hold for these more general base algebras. Also, additional properties of " $\gg "$ and “ $\perp$ " are proved in [11]. For example, under appropriate restrictions, transitivity properties such as " $M \gg N$ and $N \gg X$ imply $M \gg X$ ", and " $M \perp N$ and $N \gg X$ imply $M \perp X$ " hold for a trio $M, N, X$ consisting of a right, a two-sided, and a left module (cf. [11, Proposition 2.7]). We shall not prove these results here.

We end this section by showing how the above results can be used to attack the functional calculus problem. In fact, in the single variable case, these techniques lead to a (possibly new) proof of the standard analytic functional calculus theorem.

Let $X$ be a Banach $P$-module with the module action generated by $a \in L(X)$. If $\lambda \in C$, then $\lambda$ determines a one-dimensional $P$-module $C_{\lambda}$ with generator $\lambda$. Note that $C_{\lambda} \hat{\otimes} X=X$ and the map $\theta$ for the pair $C_{\lambda}$ and $X$ is just $(\lambda-a): X \rightarrow X$. Hence, $C_{\lambda} \perp X$ means that $\lambda-a$ is nonsingular, i.e. that $\lambda$ is in the resolvent set of the operator $a$.

Now suppose $V$ is a domain in $C$ which is contained in the resolvent set $\rho(a)$ of $a$. The space $\mathcal{O}(V) \hat{\otimes} X$ may be identified with the space of 
holomorphic $X$-value functions on $V($ cf. $[12, \S 51])$. Under this identification, the operator $\theta$ for the pair $\mathcal{O}(V), X$ is represented by the operator value function $\lambda \rightarrow(\lambda-a)$. Since $V \subset \rho(a),(\lambda-a)^{-1}$ exists on $V$ and defines an inverse for $\theta: \mathcal{O}(V) \hat{\otimes} X \rightarrow \mathcal{O}(V) \hat{\otimes} X$. We conclude that $V \subset \rho(a)$ implies $\mathcal{O}(V) \perp X$.

Now $\mathcal{O}(\boldsymbol{C})$ is the algebra of entire functions, i.e. the algebra of power series with infinite radius of convergence. It is a localization of $P$ which acts on $X$ under the representation determined by substituting $a$ for the generator in each power series. It follows from Proposition 2.2 that $\mathcal{O}(C) \gg X$.

Let $U$ be a domain containing $\operatorname{Sp}(a)=C / \rho(a)$ and set $V=\rho(a)$. Since $U \cup V=C$ it follows from Cousin's theorem (cf. $[3,1.4 .5]$ ) that the sequence

$$
0 \rightarrow \mathcal{O}(C) \stackrel{\alpha}{\rightarrow} \mathcal{O}(U) \oplus \mathcal{O}(V) \stackrel{\beta}{\rightarrow} \mathcal{O}(U \cap V) \rightarrow 0
$$

is exact, where $\alpha f=\left.\left.f\right|_{u} \oplus f\right|_{v}$ and $\beta\left(f_{1} \oplus f_{2}\right)=\left.f_{1}\right|_{U \cap V}-\left.f_{2}\right|_{U \cap V}$. Since $\mathcal{O}(\boldsymbol{C})$ is nuclear, the condition of Proposition 7.1 is satisfied. We consider $\mathcal{O}(\boldsymbol{C})$ and $\mathcal{O}(U) \oplus \mathcal{O}(V)$ to be augmented right modules with distinguished vector 1 and $1 \oplus 1$, respectively. Then we have $\mathcal{O}(\boldsymbol{C}) \gg X, \mathcal{O}(U \cap V) \perp X$ since $U \cap V \subset \rho(a)$, and hence, by Proposition 7.1, $\mathcal{O}(U) \oplus \mathcal{O}(V) \gg X$, However, $\mathcal{O}(V) \perp X$, and so Proposition 7.1 also implies that $\mathcal{O}(U) \gg X$. This means that the representation of $P$ on $X$ determined by $a$ extends to a representation of $\mathcal{O}(U)$ on $X$. This is the content of the analytic function calculus theorem in one variable.

We do not yet have nontrivial applications of the techniques of this section in the case of several free variables (although the analogous techniques for modules over the $n$-variable polynomial algebra $P_{n}$ were used in [11] to obtain a new development of the analytic functional calculus for several commuting operators). The difficulty is that we have, as yet, very little information about the family of all free analytic algebras on $n$ generators for $n>1$. For example, we have no analogue of the algebras $\mathcal{O}(U \cap V)$ and $\mathcal{O}(U \cup V)$ for a pair $\mathcal{O}(U), \mathcal{O}(V)$; in one variable these are, respectively, the minimal free analytic algebra in which $\mathcal{O}(U)$ and $\mathcal{O}(V)$ embed and the maximal free analytic algebra which embeds in both $\mathcal{O}(U)$ and $\mathcal{O}(V)$. We have no several-variable analogue of this natural lattice structure on the single variable free analytic algebras. It is not at all clear that such a lattice structure exists. Note that the algebra $\mathcal{O}(V)$ with $V=\rho(a)$, used in the above argument, is the "smallest" free analytic algebra $A$ on one generator satisfying $A \perp X$. If such a thing existed for a Banach module $X$ over $F_{n}$, its structure would undoubtedly provide nice invariants to associate with the operators $a_{1}, \ldots, a_{n}$ defining the module structure on $X$. 
One final comment: We observed that the nonsingularity of an operator $a$ on a Banach space $X$ was equivalent to $C_{0} \perp X$, where $X$ has the $P$-module structure generated by $a$ and $C_{0}$ is $C$ with the $P$-module structure generated by $0 \in \boldsymbol{C}$. Thus, for a tuple of operators $\alpha=\left(a_{1}, \ldots, a_{n}\right)$ on $X$, the relation $C_{0} \perp(X, \alpha)$ between the right $F_{n}$-module $C_{0}$ generated by $(0, \ldots, 0)$ and the left $F_{n}$-module generated by $\alpha$ might well be used as the definition of the statement that $\left(a_{1}, \ldots, a_{n}\right)$ is a nonsingular tuple of operators (in the free sense). This relation amounts to the solvability in $L(X)$ of the system of equations

$$
a_{1} b_{1}+\cdots+a_{n} b_{n}=1, \quad b_{i} a_{j}=\delta_{i j},
$$

which we discussed in Example 2 of $\S 5$.

\section{REFERENCES}

1. R. Arens and A. P. Calderón, Analytic functions of several Banach algebra elements, Ann. of Math. (2) 62 (1955), 204-216. MR 17, 177.

2. A. Grothendieck, Produits tensoriels topologiques et espaces nucléaires, Mem. Amer. Math. Soc. No. 16 (1955), 140 pp. MR 17, 763.

3. L. Hörmander, An introduction to complex analysis in several variables, Van Nostrand, Princeton, N.J., 1966. MR 34 \# 2933.

4. E. Michael, Locally multiplicatively-convex topological algebras, Mem. Amer. Math. Soc. No. 11 (1952), 79 pp. MR 14, 482.

5. H. Schaefer, Topological vector spaces, Macmillan, New York, 1966. MR 33 \# 1689.

6. R. Schatten, $A$ theory of cross-spaces, Ann. of Math. Studies, no. 26, Princeton Univ. Press, Princeton, N.J., 1950. MR 12, 186.

7. J. L. Taylor, A joint spectrum for several commuting operators, J. Functional Analysis 6 (1970), 172-191. MR 42 \#3603.

8. - The analytic-functional calculus for several commuting operators, Acta Math. 125 (1970), 1-38. MR 42 \#6622.

9. - Several variable spectral theory, Proc. Sympos. Functional Analysis (Monterey, Calif., 1969), Academic Press, New York, 1970, pp. 1-10. MR 42 \#6623.

10. Homology and cohomology for topological algebras, Advances in Math. 9 (1972), 137-182.

11. A general framework for a multi-operator functional calculus, Advances in Math. 9 (1972), 183-252.

12. F. Treves, Topological vector spaces, distributions and kernels, Academic Press, New York, 1967. MR 37 \#726.

Department of Mathematics, University of Utah, Salt lake City, Utah 84112 